\title{
The Effect of Oblique Image Acquisition on the Accuracy of Quantitative Susceptibility Mapping and a Robust Tilt Correction Method
}

\author{
Oliver C. Kiersnowski ${ }^{1}$, Anita Karsa ${ }^{1}$, Stephen J. Wastling ${ }^{2,3}$, John S. \\ Thornton $^{2,3}$, Karin Shmueli ${ }^{1}$
}

${ }^{1}$ Department of Medical Physics and Biomedical Engineering, University College London, London, United Kingdom

${ }^{2}$ UCL Queen Square Institute of Neurology, London, United Kingdom

${ }^{3}$ Lysholm Department of Neuroradiology, National Hospital for Neurology and Neurosurgery,

London, United Kingdom

Corresponding Author: Oliver C. Kiersnowski (o.kiersnowski@ucl.ac.uk)

Department of Medical Physics \& Biomedical Engineering

Malet Place Engineering Building

University College London

WC1E 7JE 


\section{Abstract}

Purpose: Quantitative susceptibility mapping (QSM) is increasingly used for clinical research where oblique image acquisition is commonplace but its effects on QSM accuracy are not well understood.

Theory and Methods: The QSM processing pipeline involves defining the unit magnetic dipole kernel, which requires knowledge of the direction of the main magnetic field $\widehat{\boldsymbol{B}}_{\mathbf{0}}$ with respect to the acquired image volume axes. The direction of $\widehat{\boldsymbol{B}}_{\mathbf{0}}$ is dependent upon the axis and angle of rotation in oblique acquisition. Using both a numerical brain phantom and in-vivo acquisitions, we analysed the effects of oblique acquisition on magnetic susceptibility maps. We compared three tilt correction schemes at each step in the QSM pipeline: phase unwrapping, background field removal and susceptibility calculation, using the root-mean-squared error and QSM-tuned structural similarity index (XSIM).

Results: Rotation of wrapped phase images gave severe artefacts. Background field removal with projection onto dipole fields gave the most accurate susceptibilities when the field map was first rotated into alignment with $\widehat{\boldsymbol{B}}_{\mathbf{0}}$. LBV and VSHARP background field removal methods gave accurate results without tilt correction. For susceptibility calculation, thresholded $k$-space division, iterative Tikhonov regularisation and weighted linear total variation regularisation all performed most accurately when local field maps were rotated into alignment with $\widehat{\boldsymbol{B}}_{\mathbf{0}}$ before susceptibility calculation.

Conclusion: For accurate QSM, oblique acquisition must be taken into account. Rotation of images into alignment with $\widehat{\boldsymbol{B}}_{\mathbf{0}}$ should be carried out after phase unwrapping and before background field removal. We provide open-source tilt-correction code to incorporate easily into existing pipelines: https://github.com/o-snow/QSM TiltCorrection.git. 


\section{Introduction}

The acquisition of oblique image slices, or an oblique slab or volume in 3D MRI, is common in clinical practice to facilitate radiological viewing of brain MRI. For example, axial slices are often aligned along the subcallosal line for longitudinal studies that require consistent repositioning of acquired images ${ }^{1}$. Alternatively, slices may be aligned perpendicular to the principle axis of the hippocampus for accurate hippocampal volume measurements and sharper hippocampal boundary delineation ${ }^{2}$. Oblique slices are also acquired to reduce image artefacts from, for example, eye motion resulting in localised blurring around the eyes and ghosting along the phase encode direction ${ }^{3}$. Note that acquiring oblique slices does not require the subject to rotate their head, as only the acquisition volume is tilted.

Quantitative susceptibility mapping ${ }^{4-6}$ utilises the information in the (conventionally discarded) phase component, $\phi(\mathbf{r})$, of the complex MRI signal from a gradient echo (GRE) sequence to calculate the tissue magnetic susceptibility, $\chi$. A typical QSM pipeline includes three key steps: 1) phase unwrapping of wraps present due to $\phi(\mathbf{r})$ being constrained to the $[-\pi, \pi)$ interval, 2) background field removal separating the local field perturbations due to internal $\chi$ sources inside the volume of interest (e.g., the brain), $\Delta B_{\text {int }}(\boldsymbol{r})$, from unwanted background field perturbations due to external sources, $\Delta B_{\text {ext }}(\boldsymbol{r})$, and 3) a local-field-to- $\chi(\mathbf{r})$ calculation to solve an ill-posed inverse problem:

$$
\Delta B_{\text {int }}(r)=\mathcal{F}^{-1}\left\{d_{z}(\boldsymbol{k}) \times \chi(\boldsymbol{k})\right\} . B_{0},
$$

$\chi(\boldsymbol{r})=\mathcal{F}^{-1}\left\{\frac{\mathcal{F} \Delta B_{\text {int }}(\boldsymbol{r})}{d_{z}(\boldsymbol{k})}\right\} \cdot B_{0}^{-1}$,

where $\mathcal{F}$ is the Fourier transform, $\mathcal{F}^{-1}$ its inverse, $B_{0}$ the magnetic field in Tesla, and $d_{z}(\boldsymbol{k})$ the $z$ component of the magnetic dipole in $k$-space $d_{z}(\boldsymbol{k})=\frac{1}{3}-\frac{k_{z}^{2}}{k^{2}}$ (see Equation 5).

Calculation of $d_{z}(\boldsymbol{k})$ requires knowledge of the ' $z$ ' direction of the main magnetic field, $\widehat{\boldsymbol{B}}_{\mathbf{0}}$, with respect to the image volume acquired. Therefore, oblique acquisition must be taken into account within the QSM pipeline otherwise incorrect $\chi$ estimates arise, as suggested by a preliminary study ${ }^{7}$ and our previous experiments ${ }^{8}$. With the increase in clinical applications of $\mathrm{QSM}^{9,10}$, accuracy in $\chi$ estimates for oblique acquisition, typical in clinical protocols, is of paramount importance in ensuring smooth translation of QSM into clinical practice. However, accurate QSM accounting for oblique acquisition is non-trivial and there are a number of techniques proposed to account for oblique acquisition in $\mathrm{QSM}^{11-14}$. The effect of these tilt correction techniques on susceptibility values has not been evaluated and it is not known at which point in the QSM pipeline these techniques should be applied. Therefore, we used a numerical phantom to carry out a comprehensive analysis of the effect of oblique acquisition on each step of the QSM pipeline, and propose three tilt correction schemes, analysing their effects on susceptibility values when applied at different points in the QSM pipeline. We also acquired several 
bioRxiv preprint doi: https://doi.org/10.1101/2021.11.30.470544; this version posted December 1, 2021. The copyright holder for this preprint (which was not certified by peer review) is the author/funder, who has granted bioRxiv a license to display the preprint in perpetuity. It is made available under aCC-BY-ND 4.0 International license.

images, in a single healthy volunteer, with volumes tilted at different angles and performed the same analysis of the effects of tilting and correction schemes in vivo. We provide open-source tilt-correction code at https://github.com/o-snow/QSM_TiltCorrection.git that uses the header information from NIfTI $^{15}$ format images to correctly orient image volumes and account for tilted acquisition for accurate QSM. 


\section{Theory}

To accurately model the magnetic dipole kernel required for the field-to- $\chi$ calculation and, in some cases, for background field removal, it is necessary to know where the magnetic field $\boldsymbol{B}_{\mathbf{0}}$ lies in acquired MRI images. Defining the two coordinate systems of interest ${ }^{7}$ as the acquired image frame $(\widehat{\boldsymbol{u}}, \widehat{\boldsymbol{v}}, \widehat{\boldsymbol{w}})$ and the scanner frame $(\widehat{\boldsymbol{x}}, \hat{\boldsymbol{y}}, \hat{\mathbf{z}})$, the main magnetic field can be written as $\boldsymbol{B}_{0, i m}$ and $\boldsymbol{B}_{0, s c}$ in the image and scanner frames, respectively:

$\boldsymbol{B}_{0, i m}=B_{0}(\hat{\mathbf{z}} . \widehat{\boldsymbol{u}}) \widehat{\boldsymbol{u}}+B_{0}(\hat{\mathbf{z}} . \widehat{\boldsymbol{v}}) \widehat{\boldsymbol{v}}+B_{0}(\hat{\mathbf{z}} . \widehat{\boldsymbol{w}}) \widehat{\boldsymbol{w}}$

$\boldsymbol{B}_{0, s c}=B_{0} \widehat{\mathbf{z}}$

In the case of non-oblique acquisition, the coordinate systems are aligned and $\boldsymbol{B}_{0, i m}=B_{0} \widehat{\boldsymbol{w}}$ in the image frame (Figure 1, left).

For the local field, $\Delta B_{\text {int }}(\boldsymbol{r})$, to $\chi(\boldsymbol{r})$ calculation (Equation 2), the magnetic dipole kernel must be calculated. Throughout this paper, references will be made to the dimensionless $k$-space dipole, $d_{z}(\boldsymbol{k})$ (Figure 1, middle row), and the dimensionless 'image-space dipole' defined in image space and Fourier transformed into k-space, $d_{z, i m}(\boldsymbol{k})$ (Figure 1, bottom row), kernels defined as follows:

$$
\begin{aligned}
& d_{z}(\boldsymbol{k})=\frac{1}{3}-\left(\widehat{\boldsymbol{B}}_{0} \cdot \widehat{\boldsymbol{k}}\right)^{2}=\frac{1}{3}-\left(\frac{k_{u}}{k}(\widehat{\mathbf{z}} \cdot \widehat{\boldsymbol{u}})+\frac{k_{v}}{k}(\hat{\mathbf{z}} \cdot \widehat{\boldsymbol{v}})+\frac{k_{w}}{k}(\widehat{\mathbf{z}} \cdot \widehat{\boldsymbol{w}})\right)^{2}, \\
& d_{z, i m}(\boldsymbol{k})=\mathcal{F}\left\{\frac{V}{4 \pi} \frac{3 \cos ^{2} \theta-1}{r^{3}}\right\},
\end{aligned}
$$

with $\widehat{\boldsymbol{k}}=k_{u} \widehat{\boldsymbol{u}}+k_{v} \widehat{\boldsymbol{v}}+k_{w} \widehat{\boldsymbol{w}}, V$ is the voxel volume, $\theta$ is the angle between $\widehat{\boldsymbol{B}}_{\mathbf{0}}$ and the $\widehat{\boldsymbol{r}}$ vector in image space where $\hat{\boldsymbol{r}}=\sqrt{\boldsymbol{u}^{2}+\boldsymbol{v}^{2}+\boldsymbol{w}^{2}}$, and $\mathcal{F}$ is the Fourier transform. It is worth noting that the image-space dipole is still in $k$-space but is defined in image-space. The periodicity of the Fourier transform constrains the boundaries of $k$-space, resulting in the dipole pattern becoming fixed along those boundaries. This causes a rotated image-space dipole to appear twisted or sheared (Figure 1, bottom row). 


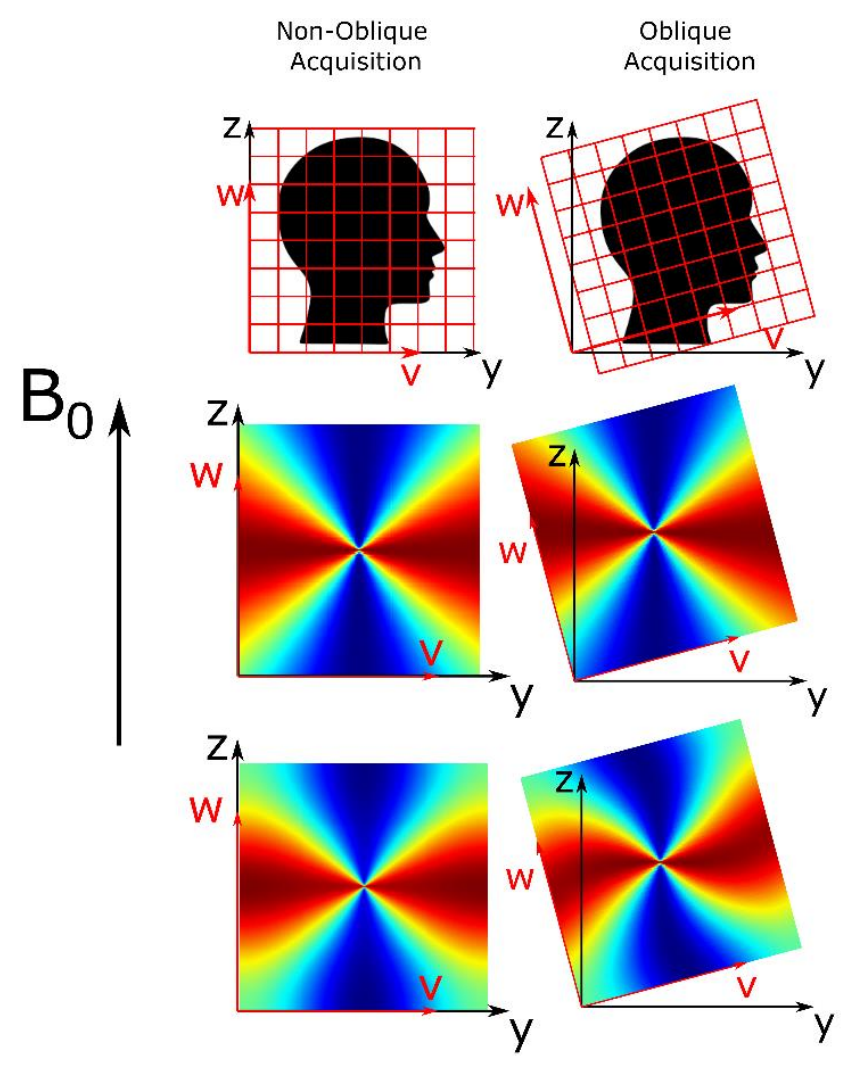

Figure 1: Non-oblique and oblique acquisition about the $\boldsymbol{x}$-axis ( $\boldsymbol{u}$-axis) of axial slices (top row) with corresponding $k$-space dipoles (middle row) and image-space dipoles (bottom row). The image axes

$(\mathbf{u}, \mathbf{v}, \mathbf{w})$ and scanner axes $(\boldsymbol{x}, \boldsymbol{y}, \boldsymbol{z})$ are shown in red and black, respectively.

\section{Methods}

It is possible to obtain the direction of $\widehat{\boldsymbol{B}}_{\mathbf{0}}$ relative to the (tilted) image axes from the image headers (e,g. DICOM or Nifti format) and, therefore, to correctly calculate the magnetic dipole kernel using either equation 5 or 6 . However, it is not clear what is the optimal method for taking oblique acquisition into account in the QSM pipeline; simply defining the dipole at an angle (see DipK or DipIm below) has been shown to be non-optimal ${ }^{7}$. We therefore investigate three proposed tilt correction schemes, and, for comparison, an uncorrected analysis pipeline (Figure 2):

1. RotPrior: rotation of the oblique image into alignment with the scanner frame prior to phase unwrapping, background field removal or the susceptibility inversion method. In this method, the dipole is defined in $k$-space in the scanner frame (using equation 5)

2. DipK: the image is left unaligned to the scanner frame and the dipole used is defined in $k$-space in the oblique image frame (using equation 5)

3. DipIm: the image is left unaligned to the scanner frame and the dipole used is defined in imagespace in the oblique image frame (using equation 6) 
4. NoRot: the oblique image is left unaligned to the scanner frame, and the $k$-space dipole is mistakenly defined in the scanner frame (using equation 4) and is thereby misaligned to the true magnetic field direction, $\widehat{\boldsymbol{B}}_{\mathbf{0}}$. This is the uncorrected method and is often the default in QSM toolboxes.

These schemes are the general names of the methods that we applied at different points in the pipeline (i.e. before phase unwrapping, background field removal, or susceptibility calculation) and for different methods or algorithms. For example, as no dipole kernel is necessary for phase unwrapping (and we substitute the dipole operations illustrated in Figure 2 with $\widehat{\boldsymbol{B}}_{\mathbf{0}}$-orientation-independent unwrapping operations), we have called the only two schemes appropriate before unwrapping RotPrior and NoRot where the image volume is rotated prior to unwrapping and after, respectively.

All rotations were carried out about the $x$-axis ( $u$-axis) to simulate single oblique acquisition, the $y$-axis ( $v$-axis) for confirmation, and about the $y=x$ axis ( $v=u$ axis) to simulate double oblique acquisition. Rotations were undertaken using FSL FLIRT ${ }^{16}$ with trilinear interpolation. To facilitate comparisons, all images left in the image-frame after correction (DipK, DipIm and NoRot) were rotated back into alignment with the scanner axes (see black arrow in Figure 2). Unless stated otherwise, all processing and analysis operations were carried out using MATLAB (MathWorks Inc., Natick, MA, USA). 


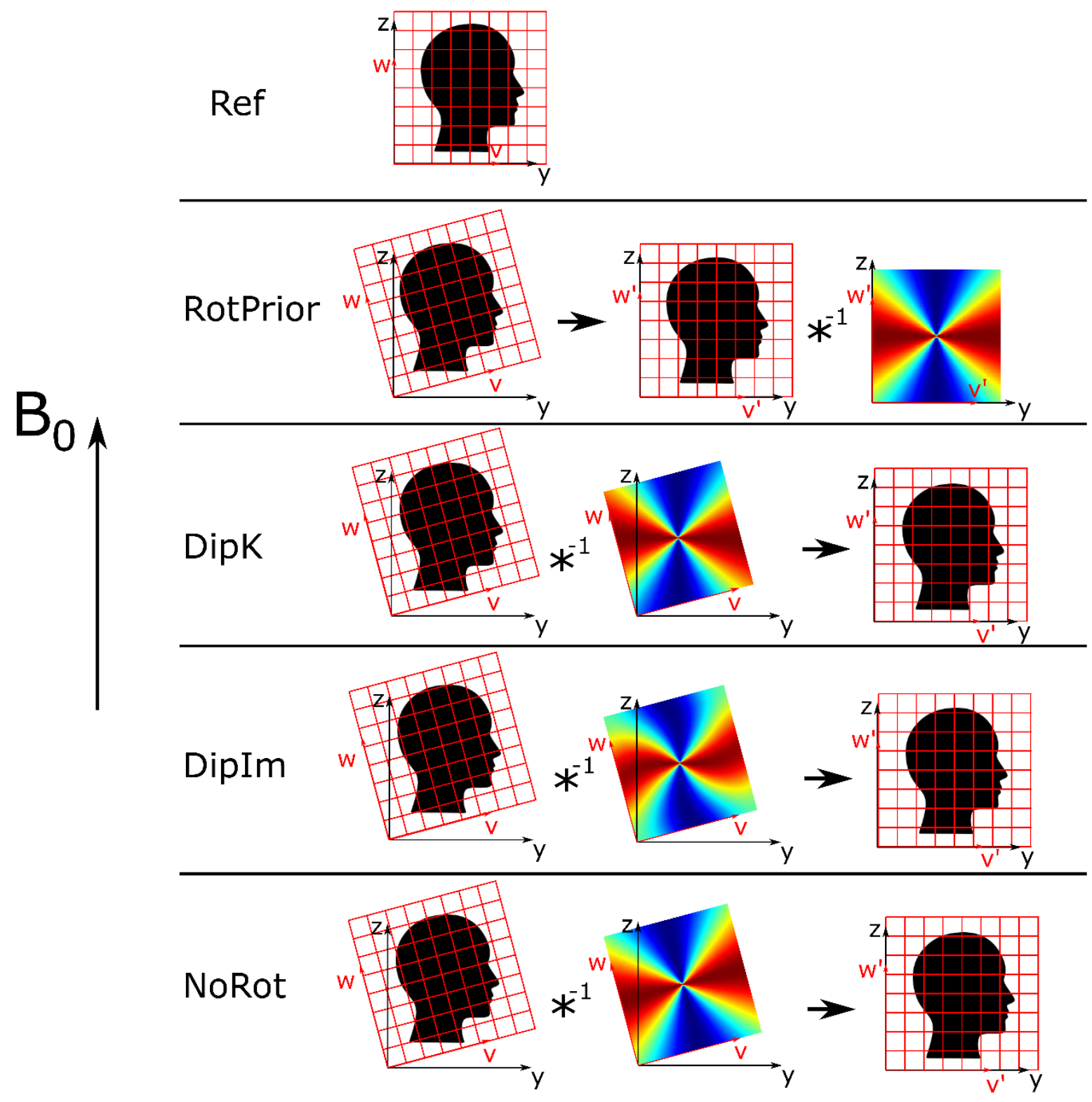

Figure 2: All tilt correction schemes including the reference, non-oblique acquisition for rotations about the $x$-axis. The native (oblique) image space $(\widehat{\boldsymbol{u}}, \widehat{\boldsymbol{v}}, \widehat{\boldsymbol{w}})$ was transformed to $\left(\widehat{\boldsymbol{u}}^{\prime}, \widehat{\boldsymbol{v}}^{\prime}, \widehat{\boldsymbol{w}}^{\prime}\right)$ aligned with the scanner frame. The black arrow denotes rotation into the scanner frame of reference. DipK,

DipIm and NoRot were rotated back into the reference (scanner) frame post-correction to facilitate comparisons. RotPrior and NoRot still apply when no dipole is used.

\section{Numerical Phantom Investigations}

Multi-echo ( $T E=4,12,20,28 \mathrm{~ms}$ ) magnitude and phase images, from a numerical phantom ${ }^{17}$, with (originally) no phase wraps or background fields present, were used to independently investigate the effect of the three tilt correction methods (described above), and no correction, on each step in the QSM pipeline. 
We carried out these investigations with two image volumes: one unpadded with the original matrix size $164 \times 205 \times 205$ and a second volume padded to $357 \times 357 \times 357$. The padded matrix size was chosen as the long diagonal of the initial volume (padded to a cube: $205 \times 205 \times 205$ ) and rounded up to the nearest odd integer. This was to ensure that none of the original frequency coefficients of the unit dipole field were cut off due to rotations about any of the three axes. An odd matrix size meant that there was a true centre of rotation correctly located within a single central voxel.

\section{Phantom: Susceptibility Calculation}

Local field maps, $\Delta B_{\text {int }}(\boldsymbol{r})$, were calculated from a non-linear $\mathrm{fit}^{18}$ over all echo times (for the most accurate field estimates ${ }^{19}$ ) of the complex data set created by combining the magnitude and background field-free phase images. These local field maps, obtained from the supplied raw phantom data, were free of any (synthetic) background fields or phase wraps, and therefore allowed investigation of the effect of oblique acquisition on $\chi$ calculation alone. To simulate oblique acquisition, local field maps were rotated between $\pm 45^{\circ}$ in $5^{\circ}$ increments. All tilt correction methods described (and no correction) were compared for three $\chi$ calculation methods chosen to cover the two main approaches: direct noniterative solutions (in $k$-space) and iterative solutions (in image-space).

The first method tested was direct, thresholded $k$-space division (TKD $)^{20,21}$ where a modified dipole kernel was generated in $k$-space with values below a threshold, $\delta=2 / 3$, replaced by the signed threshold value:

$d_{z}^{\prime}(\delta, k)=\left\{\begin{array}{c}d_{z}(\delta, \boldsymbol{k}) \text { if }\left|d_{z}(\delta, \boldsymbol{k})\right|>\delta \\ \operatorname{sign}\left(d_{z}(\delta, \boldsymbol{k})\right) \cdot \delta \quad \text { otherwise }\end{array}\right.$

The dipole was originally defined according to DipK and DipIm and then always thresholded in $k$-space. Susceptibility underestimation was corrected by multiplication with a correction factor, $c_{\chi}(\delta)$, calculated according to $^{22}$.

The second and third $\chi$ calculation methods aim to iteratively solve for $\chi$ through the minimisation of

$\underset{\chi}{\arg \min }\left\|M W\left(\Delta B_{\text {int }}-B_{0} \cdot\left(d_{z} * \chi\right)\right)\right\|_{2}^{2}+R(\chi)$

where $M$ is a binary mask, $W$ is a weighting term and $R(\chi)$ is the data regularisation term that reflects some prior information about $\chi$. Iterative Tikhonov regularisation ${ }^{23,24}$ was chosen as it has performed well in a variety of QSM applications including outside the brain ${ }^{25-27}$. It was applied with $R(\chi)=\alpha\|\chi\|_{2}^{2}$, a regularisation parameter $\alpha=0.003$ (chosen through an L-Curve analysis ${ }^{28}$ ), and $\mathrm{W}$ reflecting the spatially varying noise, and was also corrected for $\chi$ underestimation $^{22}$. Weighted linear total variation 
regularisation (from the FANSI toolbox ${ }^{29,30}$ ) with $R(\chi)=\alpha|\nabla \chi|_{1}, \alpha=6.31 \times 10^{-5}$ (chosen through an L-Curve analysis) and $\mathrm{W}$ the magnitude of the complex data ${ }^{30}$ was also tested. This method was chosen as total variation based iterative approaches were shown to produce the most accurate susceptibility maps in the 2019 QSM challenge $2.0^{31}$.

Mean $\chi$ values were calculated in five deep gray matter regions of interest (ROIs): the caudate nucleus, globus pallidus, putamen, thalamus and red nucleus. All susceptibility maps were compared using the RMSE and XSIM ${ }^{32}$ metrics relative to the supplied ground truth susceptibility map at $0^{\circ}$.

\section{Phantom: Background Field Removal}

For the background field removal step, local field maps from the numerical phantom required the addition of synthetic background fields, which were then removed following the three different tiltcorrection methods (and no correction). After background field removal, the susceptibility maps were calculated from the resulting field maps using the $\chi$ calculation method found to be optimal in the above described assessment.

To investigate the effect of tilt correction schemes on the background field removal step, synthetic background fields, $\Delta B_{\text {ext }}(\boldsymbol{r})$ (Figure 3, bottom left), were added to the local field maps used in the Methods: Phantom: Susceptibility Calculation section. The background fields were calculated using the forward model, i.e. through a convolution, formulated as a multiplication in Fourier space, between the unit magnetic dipole field and a head-shaped susceptibility map ${ }^{33,34}$ :

$\Delta B_{\text {ext }}(\boldsymbol{r})=\mathcal{F}^{-1}\left\{\chi_{\text {head }}(\boldsymbol{k}) \cdot d_{z}(\boldsymbol{k})\right\}$.

$\chi_{\text {head }}$ is the head-shaped susceptibility map, with soft tissue (-9.4ppm) and bone (-11.4ppm) $)^{7,35}$ regions obtained by thresholding the magnitude (sum of squares over all echoes) and a pseudo-CT ${ }^{36-38}$ image, respectively (Figure 3). The magnitude and pseudo-CT images were padded from their original matrix size of $164 \times 205 \times 205$ to $512 \times 512 \times 512$ to ensure edge effects from the periodic Fourier transform were minimised around the volume of interest. These synthetic background fields were then cropped back to their original matrix size and added to the local field maps obtained previously simulating a total field map, $\Delta B(\boldsymbol{r})=\Delta B_{\text {int }}(\boldsymbol{r})+\Delta B_{\text {ext }}(\boldsymbol{r})$. To simulate oblique acquisition, total field maps were rotated between $\pm 45^{\circ}$ in $5^{\circ}$ increments. 
bioRxiv preprint doi: https://doi.org/10.1101/2021.1130.470544; this version posted December 1, 2021. The copyright holder for this preprint (which was not certified by peer review) is the author/funder, who has granted bioRxiv a license to display the preprint in perpetuity. It is made available under aCC-BY-ND 4.0 International license.

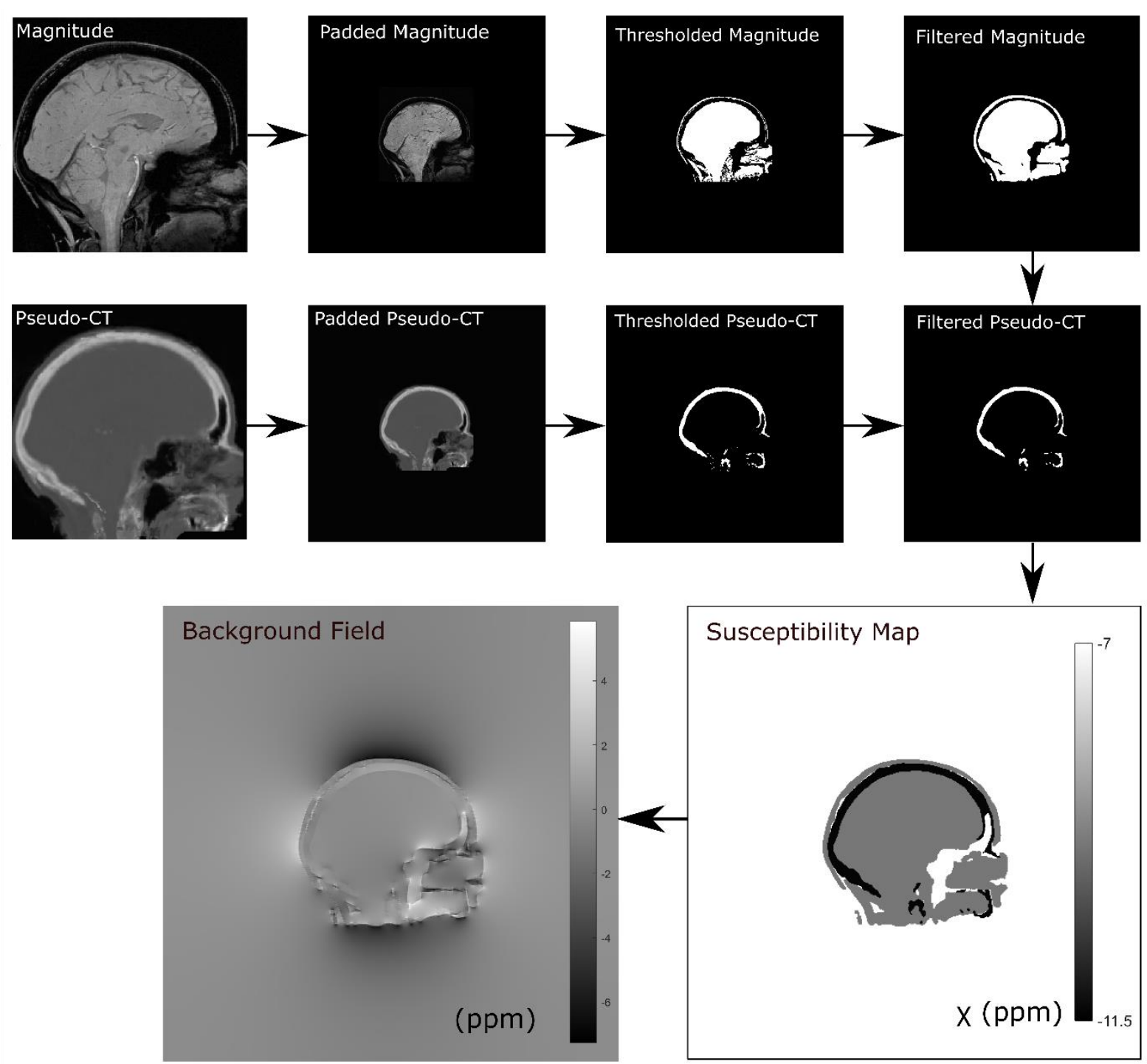

Figure 3: Method for calculating the synthetic background field from a head-shaped susceptibility map obtained by thresholding the phantom magnitude image and a pseudo-CT image to delineate soft tissue and bone, respectively. The thresholded magnitude and pseudo-CT images were filtered for smoothness using a $3 \times 3 \times 3$ box-filter.

Three different state-of-the-art background field removal methods ${ }^{39}$ were then used to remove the synthetic background fields, $\Delta B_{\text {ext }}(\boldsymbol{r})$, from the tilted total field maps. Projection onto dipole fields $(\mathrm{PDF})^{40}$ was used following tilt correction with all three correction schemes and no correction because PDF is orientation dependent, i.e. it uses the dipole field from $d_{z}(\boldsymbol{k})$ (Equation 5). Laplacian boundary value $(\mathrm{LBV})^{41}$ and variable-kernel sophisticated harmonic artifact reduction for phase data (VSHARP) $)^{42}$ were tested with RotPrior and NoRot only, as LBV and V-SHARP are orientation independent methods, i.e. they do not use the dipole field. Following rotation back into the reference frame (equivalent to RotPrior for the susceptibility calculation step), susceptibility maps were calculated from all local field maps using iterative Tikhonov regularisation (regularisation parameter 
$\alpha=0.003$ ) as this was found to be optimal. Susceptibility maps were compared using the root mean squared error (RMSE) and the QSM-tuned structural similarity index (XSIM) ${ }^{32}$ relative to the ground truth susceptibility map at $0^{\circ}$.

\section{Phantom: Phase Unwrapping}

To investigate the effect of tilt correction on phase unwrapping, the synthetic background fields added in the previous section (Methods: Phantom: Background Field Removal) induced phase wraps when the phase was constrained to the $[-\pi, \pi)$ interval, which were then unwrapped. Susceptibility maps were then calculated from these unwrapped field maps using background field removal and susceptibility calculation algorithms found to be optimal in the experiments described in the previous sections (Methods: Phantom: Susceptibility Calculation and Background Field Removal).

To investigate the effect of tilt correction on phase unwrapping, phase wraps were introduced into the wrap-free phantom images via the additional synthetic background field described above. From each total field map at each angle, $\Delta B(\boldsymbol{r})=\Delta B_{\text {int }}(\boldsymbol{r})+\Delta B_{\text {ext }}(\boldsymbol{r})$, multi-echo unwrapped phase images were simulated via scaling the tilted total field maps at each echo time according to $\phi(\boldsymbol{r}, T E)=\gamma$. $T E \cdot \Delta B(\boldsymbol{r})$.

At every tilt angle, a complex data set $(S)$ was made from the multi-echo magnitude images $(M)$ and simulated phase images $(\phi)$ using $S=M(r, T E) e^{i \phi(r, T E)}$, which constrained the phase to the range $[-\pi, \pi)$, resulting in phase wraps. A wrapped total field map was calculated via a non-linear fit over all echo times ${ }^{18}$, which then underwent phase unwrapping using the commonly used Laplacian ${ }^{43}$ and SEGUE ${ }^{44}$ techniques with the NoRot and RotPrior tilt correction methods. After rotating all the unwrapped images back into the reference frame (equivalent to RotPrior for the background field removal step and susceptibility calculation step), susceptibility maps were then calculated with PDF $^{40}$ background field removal and susceptibility calculation using iterative Tikhonov regularisation (regularisation parameter $\alpha=0.003$ ) as we found these to provide optimal results.

\section{Investigations In Vivo}

\section{In Vivo: MRI Acquisition}

3D gradient-echo brain images of a healthy volunteer were acquired on a 3T Siemens Prisma-Fit MR system (National Hospital for Neurology and Neurosurgery, London, UK) using a 64-channel head coil across a range of image volume orientations. Note that the volunteer did not tilt their head but remained in the same position throughout the experiment. The image volume was tilted about the $x$-axis, as this is the most common in clinical practice, from $-20^{\circ}$ to $+20^{\circ}$ in $5^{\circ}$ increments, with the reference image at $0^{\circ}$ representing a non-oblique acquisition. Each image volume was acquired in $3 \min 23 \mathrm{~s}$ with $\mathrm{TR}=$ $30 \mathrm{~ms}$; TEs $=4.92,9.84,14.76,19.68,24.60 \mathrm{~ms} ; 1.23 \mathrm{~mm}$ isotropic voxels; FOV $=256 \times 192 \times 216.6$ mm; Matrix Size $=208 \times 156 \times 176$; bandwidth $=280 \mathrm{~Hz} /$ pixel; flip angle $15^{\circ} ; 6 / 8$ partial Fourier 
along PE1 and PE2; ASPIRE coil combination ${ }^{45}$; and GRAPPAPE1 acceleration $=3$ (FE direction: A >>P, PE1 direction: R>>L, PE2 direction F>>H).

\section{In Vivo: Phase Unwrapping}

For all angles/volumes, a total field map and a noise map were obtained using a non-linear fit of the complex data ${ }^{46}$ from the MEDI toolbox ${ }^{14}$. A brain mask was created using the brain extraction tool $(B E T)^{47}$ with default settings applied to the final echo magnitude image for a conservative brain mask estimate. As with the numerical phantom, both the RotPrior and NoRot correction schemes were applied. Residual phase wraps were then removed using Laplacian unwrapping ${ }^{43}$ and SEGUE ${ }^{44}$. To investigate the effect of the correction schemes on this step in the pipeline, unwrapped total field maps were rotated back into the reference frame (equivalent to RotPrior for the background field removal step and susceptibility calculation step) and susceptibility maps were created using PDF background field removal and susceptibility calculation with iterative Tikhonov regularisation ( $\alpha=0.017$ chosen through an L-Curve analysis).

As in the numerical phantom, and also due to very slight unavoidable changes in subject position between scans, the unwrapped field maps and susceptibility maps were registered into the reference image space to facilitate comparisons of results in vivo. To carry out this registration, the magnitude image (added in quadrature over all echoes) for each angle was rigidly registered to the $0^{\circ}$ magnitude using NiftyReg ${ }^{48}$ resulting in a transformation matrix per angle/volume, which was applied to bring all angles/volumes into the same common reference space.

\section{In Vivo: Background Field Removal}

The Laplacian unwrapped field maps described above (in Methods: In Vivo: Phase Unwrapping) for volumes at all angles, and prior to any registrations or rotations, were used to investigate the effect of oblique acquisition on background field removal. As for the numerical phantom, for each field map, at each angle, background fields were removed using $\mathrm{PDF}^{40}$ with all tilt correction schemes and no correction, and using $\mathrm{LBV}^{41}$ and V-SHARP ${ }^{42}$ with only RotPrior and NoRot. For all three background field removal methods, the brain mask was eroded by 4 outer voxels ${ }^{49}$. RotPrior was performed twice: with mask erosion either before or after the rotation to compare the effects of interpolation, particularly along the boundaries of the field map on PDF and V-SHARP, as it is known that boundary effects arise in these background field removal methods ${ }^{39}$.

For comparison purposes, after rotation and registration of the local field maps back into the reference frame (equivalent to RotPrior for the susceptibility calculation step), susceptibility maps were calculated from the local field maps using iterative Tikhonov regularisation $(\alpha=0.017$, chosen with an L-Curve). Local field maps and susceptibility maps were compared with RMSE and XSIM metrics (XSIM only for the susceptibility maps) relative to the $0^{\circ}$ reference image. 


\section{In Vivo: Susceptibility Calculation}

To investigate the effect of oblique acquisition on the $\chi$ calculation step in the pipeline, we used the local field maps following Laplacian unwrapping and LBV background field removal (described in the previous section Methods: In Vivo: Background Field Removal), prior to any registrations or rotations. LBV was chosen as we found it to be relatively robust to oblique acquisition, thereby allowing our analysis to focus on the effect of the $\chi$ calculation step alone. The three tilt correction schemes (and no correction) were compared using the same three $\chi$ calculation methods as for the numerical phantom: TKD, iterative Tikhonov regularisation with a regularisation parameter $\alpha=0.017$ from an L-curve analysis, and weighted linear TV with a regularisation parameter $\alpha=2 \times 10^{-4}$ found also from an Lcurve (larger than for the phantom due to increased noise in vivo).

The resulting susceptibility maps were transformed into the reference space as described in the earlier section Methods: In Vivo: Phase Unwrapping. The same ROIs as in the numerical phantom were investigated and were obtained by registering the $\mathrm{EVE}^{50}$ magnitude image with the reference magnitude image (at the first echo time) and applying the resulting transformation to the EVE ROIs. Mean $\chi$ values were calculated in these ROIs for all tilt angles and all correction schemes. RMSE and XSIM metrics were also used to compare the susceptibility maps from the different angles and tilt correction schemes. 


\section{Results}

\section{Numerical Phantom}

All numerical phantom results shown here are for rotations of image volumes about the $x$-axis with unpadded matrices. Note that acquisitions tilted about the $y$-axis and $y=x$ axis as well as images with padded matrices all gave similar results (see Supporting Figures 1-3). We chose to display these results as the padded matrix size leads to increased computation time, which is not recommended in a practical setting, and the $x$-axis is the most common axis of rotation for oblique acquisition.

When wrapped phase images are rotated prior to phase unwrapping with the correction scheme RotPrior, artefacts arise for both Laplacian and SEGUE unwrapping (Figure 4). SEGUE appears to fail with RotPrior as it incorrectly identified phase wraps and thereby removed a portion of the mask (Figure $4 c)$.

When using PDF for background field removal, RotPrior is the most accurate method, and the largest errors arise from DipIm and NoRot (Figure 5). Striping artefacts are present in the local field map from the DipK method. LBV and V-SHARP are shown to be largely unaffected by oblique acquisition.

Figure 6 summarises the mean susceptibility in the Caudate Nucleus and Thalamus, alongside the RMSE and XSIM measurements across all angles for all three $\chi$ calculation methods. TKD and iterative Tikhonov methods are most accurate with RotPrior, and least accurate when the dipole is misaligned to the main magnetic field (NoRot). Weighted linear TV is relatively robust to oblique acquisition with RotPrior and DipK performing similarly. However, DipK shows more variability in $\chi$ at the ROI level than RotPrior. Weighted linear TV with DipIm fails at non-zero angles and NoRot results in the largest errors. Example susceptibility maps are shown in Figure 7, highlighting the widespread $\chi$ errors that arise when the magnetic dipole is defined incorrectly (NoRot). 


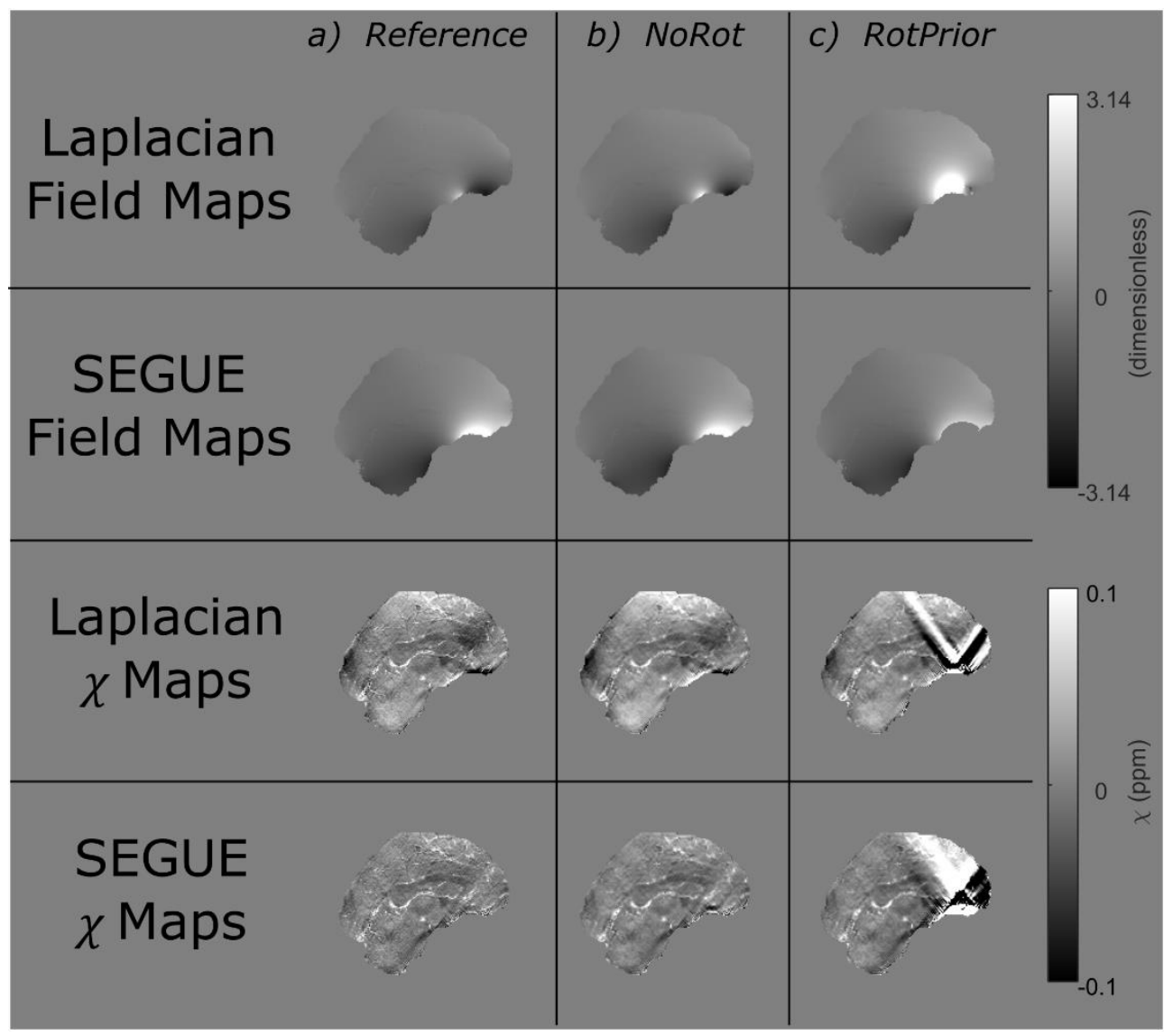

Figure 4: The effect of tilt correction before phase unwrapping in the numerical phantom. Phase unwrapped field maps and the resulting susceptibility maps at $15^{\circ}$ for the NoRot (column b) and RotPrior (column c) tilt correction methods relative to the reference (column a). Rotation of the wrapped field maps prior to phase unwrapping with both Laplacian and SEGUE techniques results in errors along phase wraps and incorrect unwrapping, leading to prominent artefacts in the final susceptibility maps. 

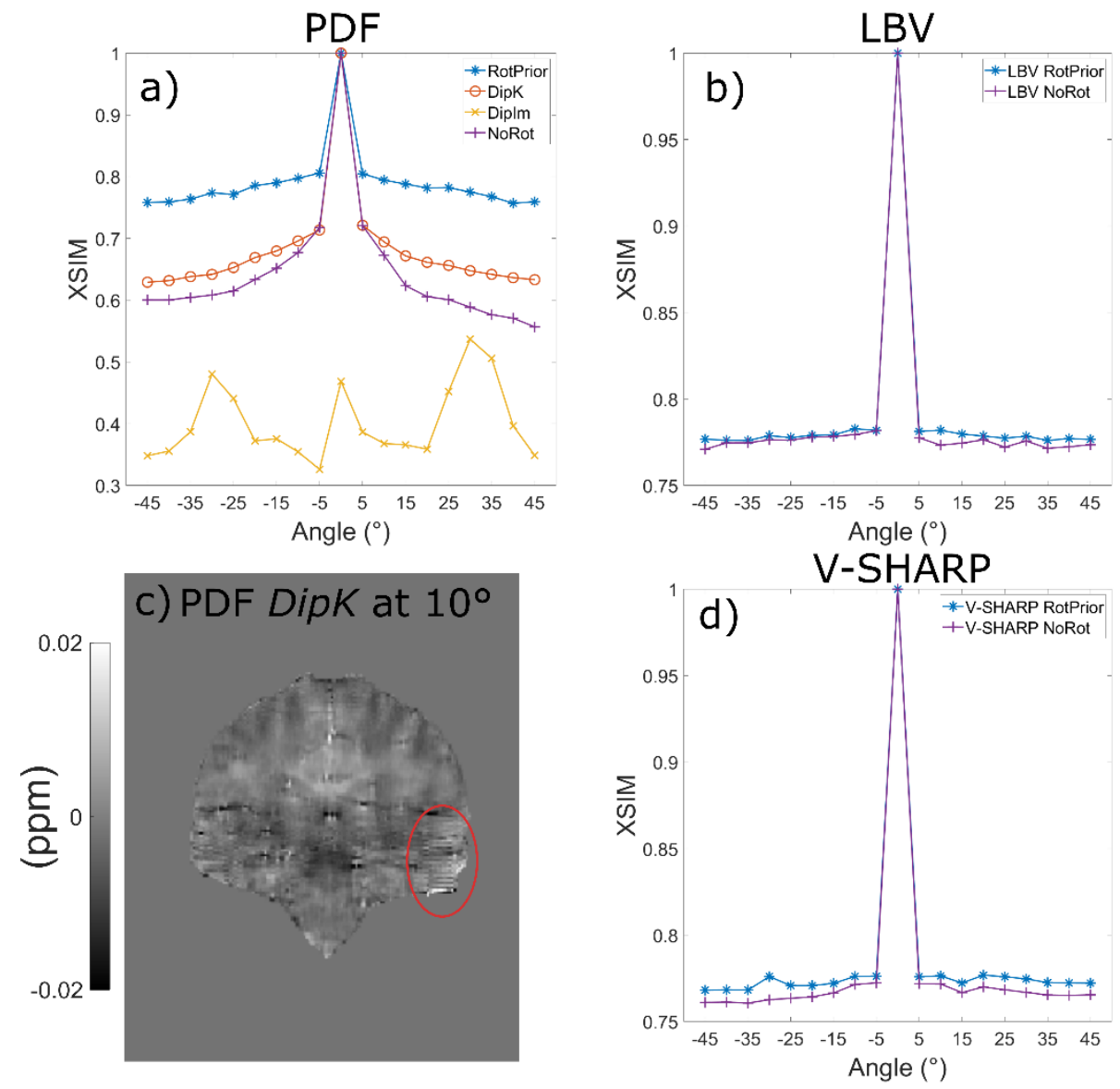

Figure 5: The effect of different tilt correction schemes on QSM with three background field removal methods in a numerical phantom. For PDF (a) the XSIM metric shows that RotPrior gives the most accurate susceptibilities, with DipIm performing the worst. When using PDF with DipK, striping artefacts (c, red ellipse) arise in the local field maps for tilted acquisitions. LBV and V-SHARP (b, d) are shown to be largely unaffected by oblique acquisition with differences arising primarily from rotation interpolations. RMSE measurements (not shown) followed the same pattern as the XSIM metric. 

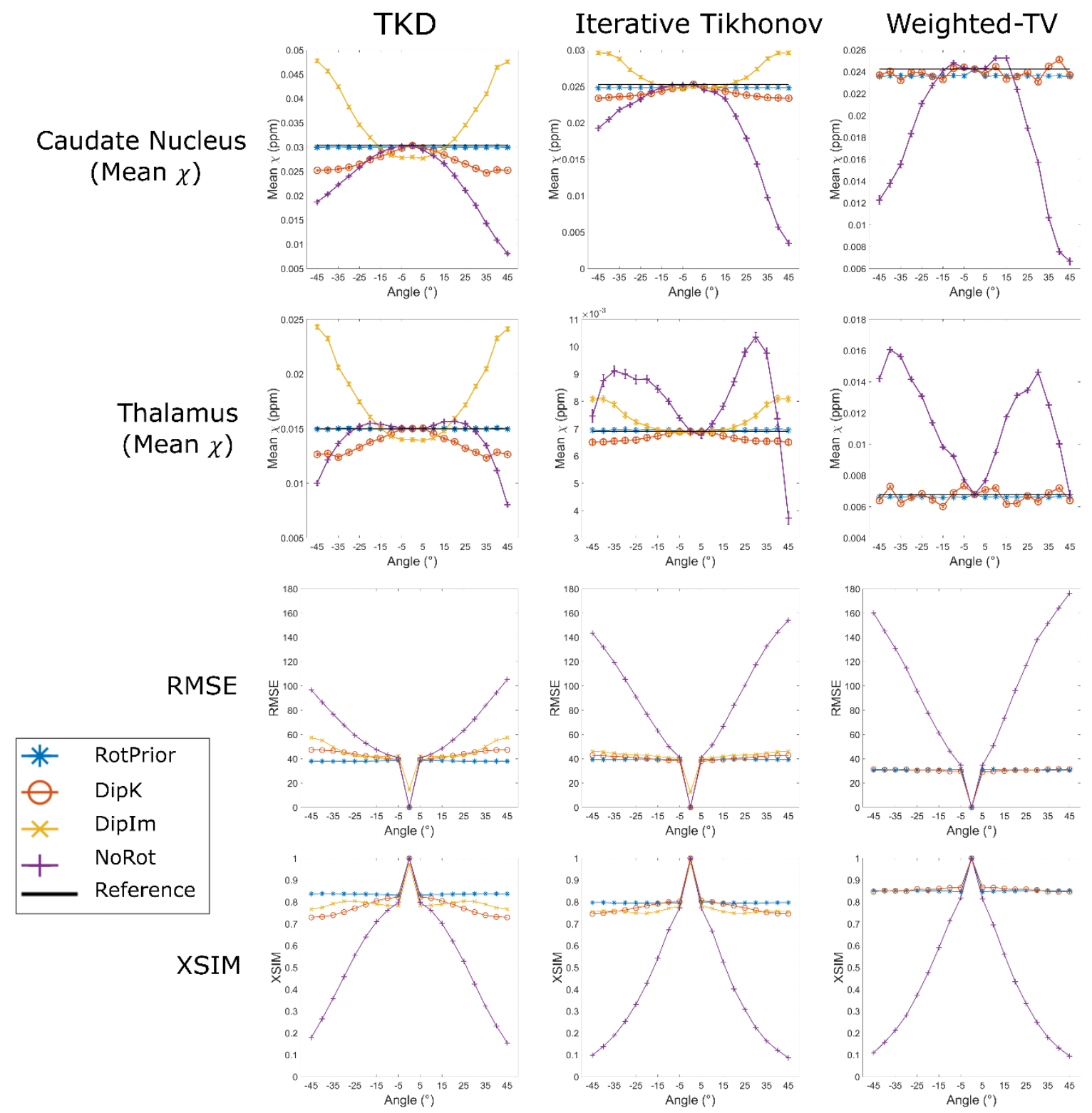

Figure 6: Mean susceptibilities in the caudate and thalamus (top rows), and RMSE and XSIM (bottom rows) across all tilt angles for all tilt correction schemes and all three $\chi$ calculation methods in the numerical phantom. NoRot performs worst across all angles. RotPrior is the most accurate tilt correction scheme. For weighted linear TV, DipK and RotPrior have similar XSIM values but the mean thalamus $\chi$ varies more over angles with DipK. Note that DipIm is not shown for wITV as this method fails. 


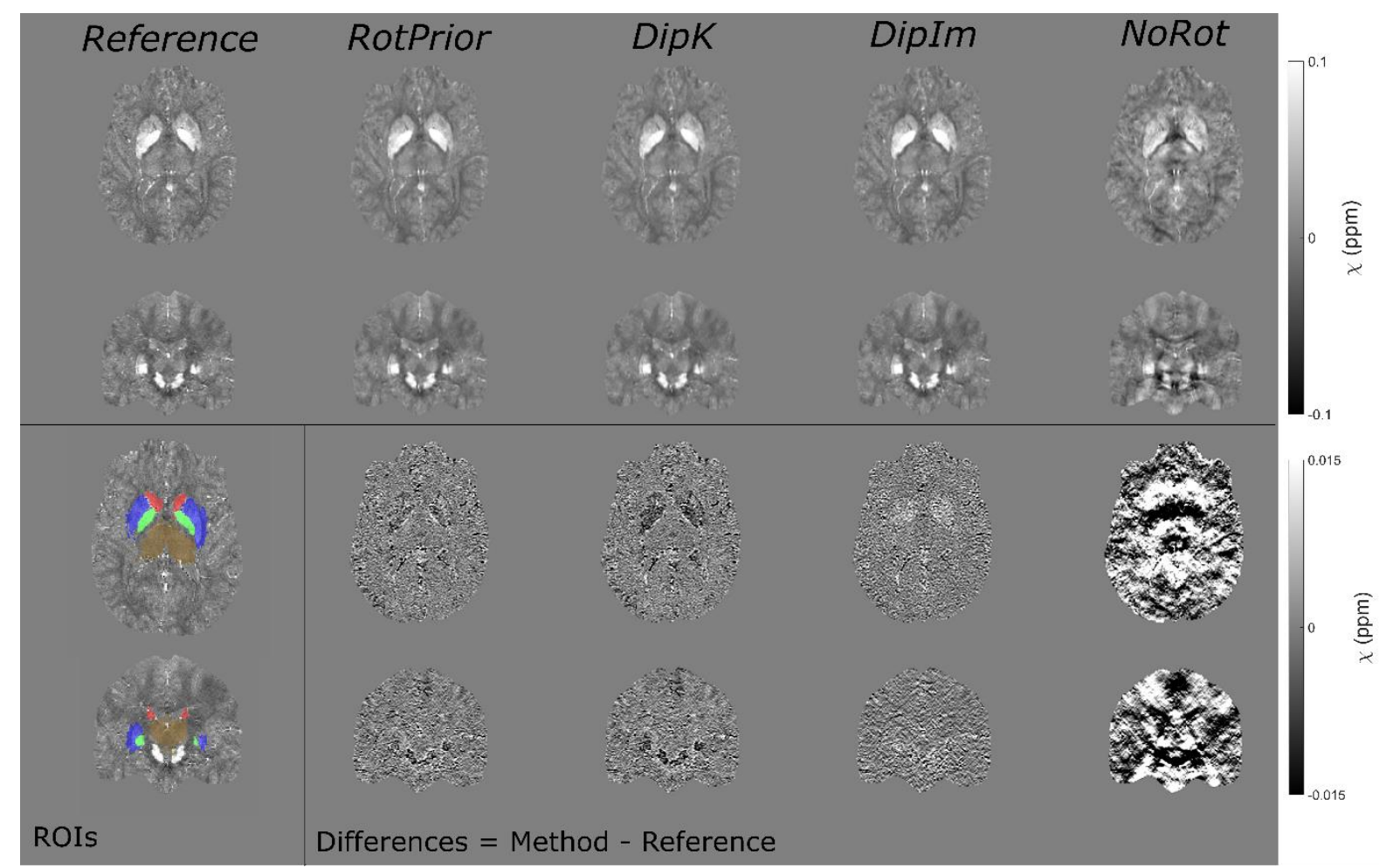

Figure 7: $\chi$ maps and difference images illustrating the effects of all tilt correction schemes in the numerical phantom. An axial and a coronal slice are shown for a volume tilted at $25^{\circ}$ and a reference $0^{\circ}$ volume with all $\chi$ maps calculated using the iterative Tikhonov method. The ROIs analysed are also shown (bottom left). RotPrior performs the best while NoRot results in substantial $\chi$ errors across the whole brain. The results from TKD and weighted-TV (not shown) are very similar.

\section{In Vivo}

In vivo, Laplacian and SEGUE phase unwrapping with RotPrior have the same image artefacts as in the numerical phantom (not shown here) compared with NoRot, with incorrect identification of phase wraps when wrapped field maps are rotated prior to phase unwrapping.

Figure 8 shows that PDF background field removal is most accurate with RotPrior and least accurate with DipIm followed by NoRot, confirming the results obtained in the phantom (Figure 5). Striping artefacts are present in the DipK method for PDF in the local field maps prior to re-orientation for comparison purposes (Fig. 8c). Rotation interpolation obscures these artefacts in the in-vivo images. LBV and V-SHARP are shown to be largely orientation independent in the in vivo case as expected.

When RotPrior was performed with mask erosion before rotating the total field map, artefacts arose along the boundaries of the PDF local field map (See Supporting Figure 4). PDF performs more robustly if mask erosion is carried out after rotation, whereas V-SHARP appears to perform equally well in both scenarios.

Figure 9 shows the effect of all tilt correction schemes on susceptibility calculation in vivo and confirms that NoRot results in the largest susceptibility errors and that RotPrior is comparable to DipK 
between $\pm 20^{\circ}$. At non-zero tilt angles, RMSE and XSIM have a respectively high/low baseline level arising from rotation and registration interpolations. Both RotPrior and DipK perform better than DipIm, in agreement with results in the phantom (Figure 6). Difference images (Figure 10) also confirm those obtained in the numerical phantom (Figure 7). Subtle effects found in several of the phantom ROIs (Figure 6) were not apparent in vivo (Figure 10).
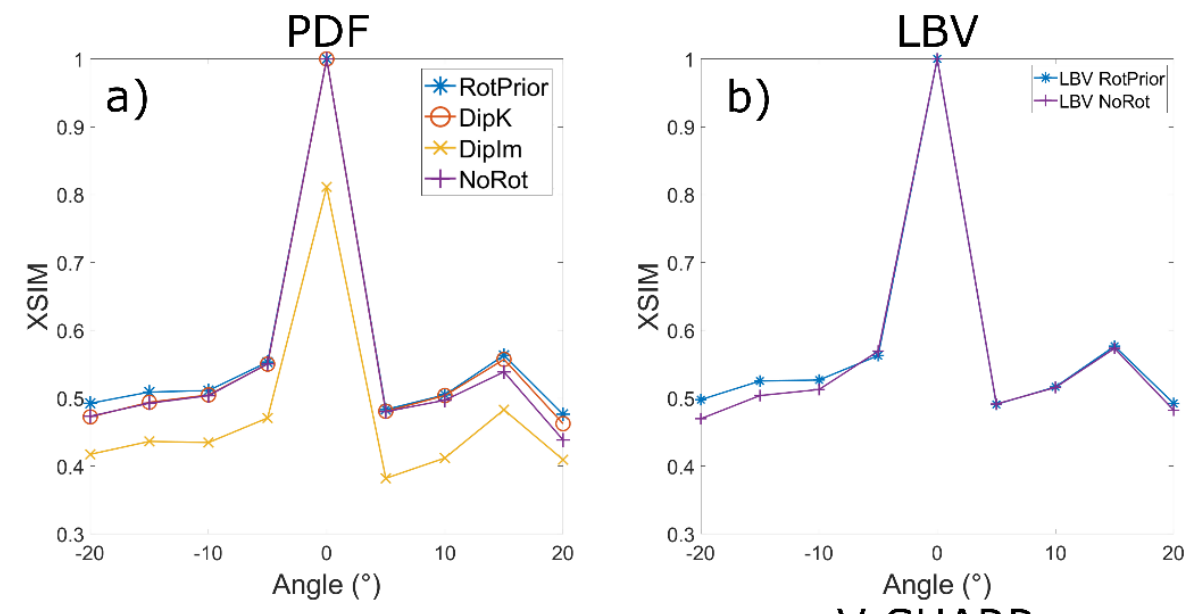

\section{c) PDF DipK at $-10^{\circ}$}
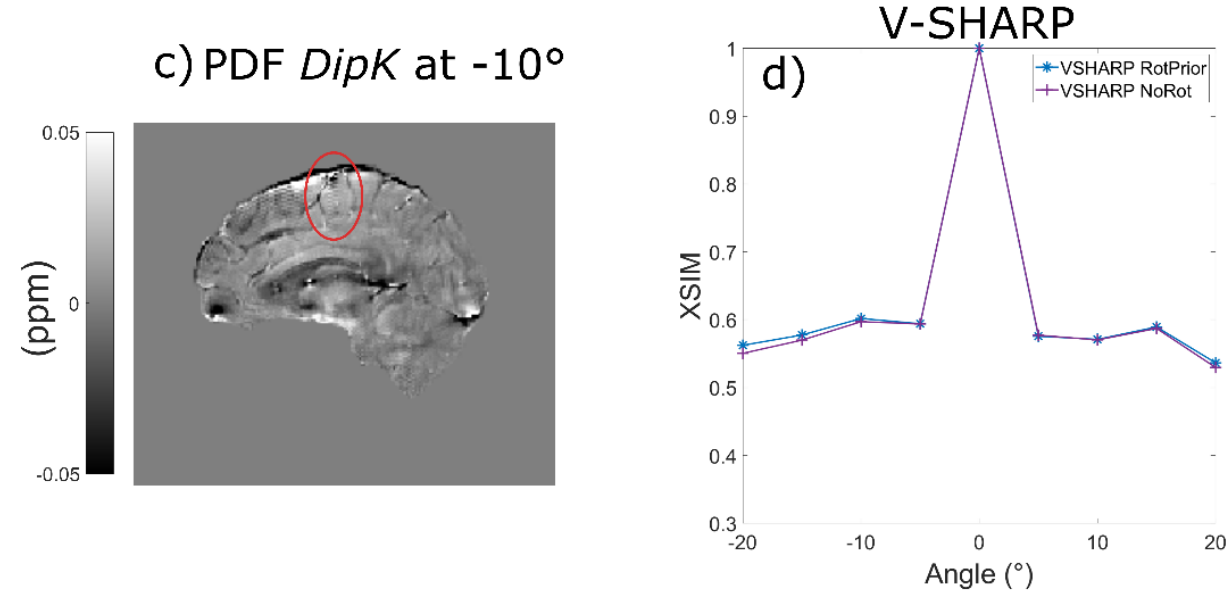

Figure 8: The effect of different tilt correction schemes on background field removal in vivo. XSIM measurements were used to compare susceptibility map quality calculated with iterative Tikhonov regularisation after background field removal. PDF (a) works most accurately with RotPrior and least accurately with DipIm, followed by NoRot, confirming results in the phantom (Figure 5). Striping artefacts are found in local field maps for DipK when using PDF (c, red ellipse) but are obscured after rotation and registration back into the reference $0^{\circ}$ space due to interpolation. LBV (b) and V-SHARP (d) are shown to be unaffected by oblique acquisition in vivo as well as in the numerical phantom (Figure 5d). 


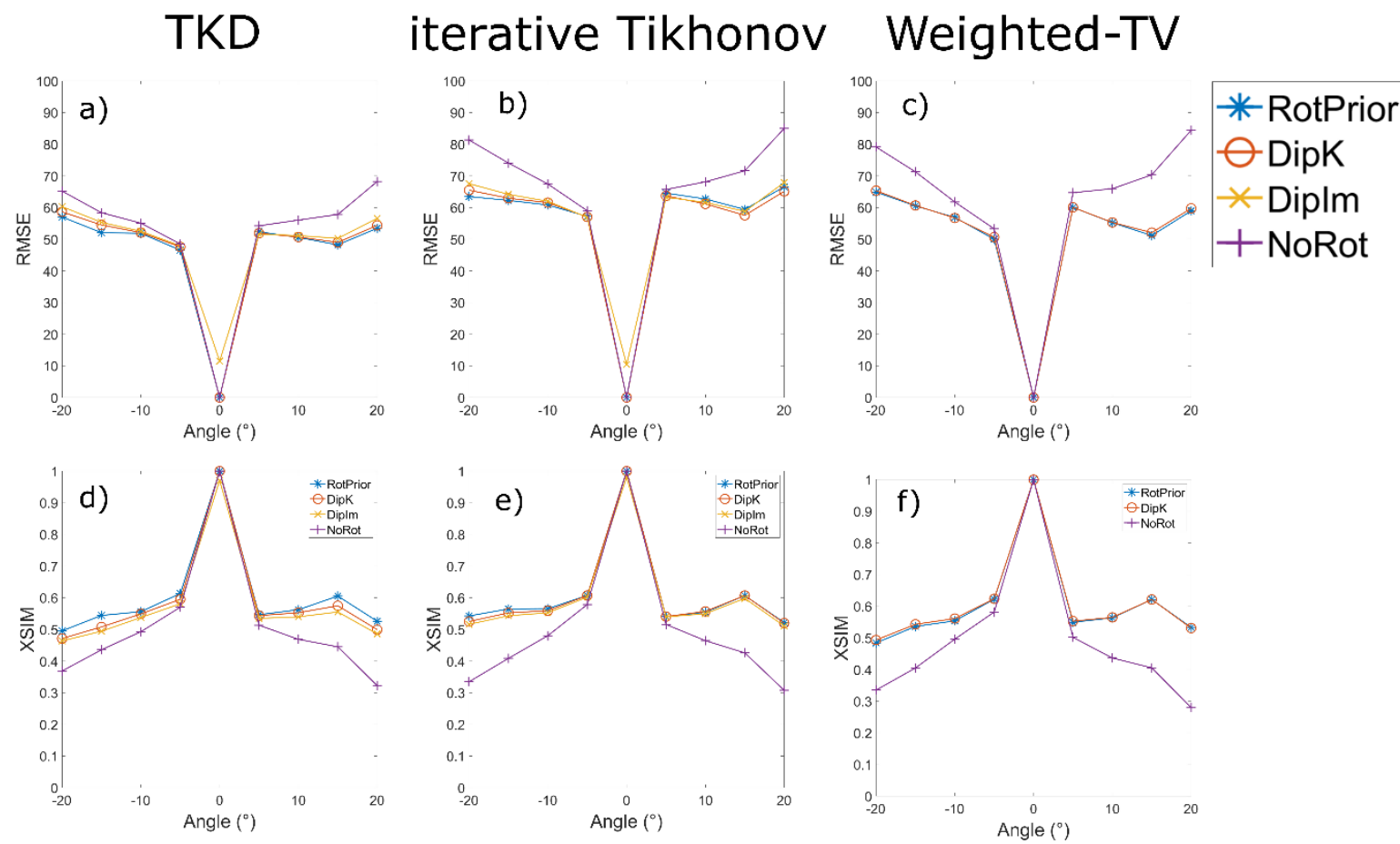

Figure 9: RMSE and XSIM plots over all angles for all tilt correction schemes and all three $\chi$ calculation methods in one subject in vivo. These results are similar to those in the numerical phantom (Figure 3) with RotPrior performing best and NoRot performing worst across all methods. At nonzero tilt angles, RMSE and XSIM have a respectively high/low baseline level arising from rotation and registration interpolations. DipIm fails for weighted linear TV and is, therefore, omitted from the plots in the last column. 
bioRxiv preprint doi: https://doi.org/10.1101/2021.11.30.470544 t this version posted December 1, 2021. The copyright holder for this preprint (which was not certified by peer review) is the author/funder, who has granted bioRxiv a license to display the preprint in perpetuity. It is made available under aCC-BY-ND 4.0 International license.

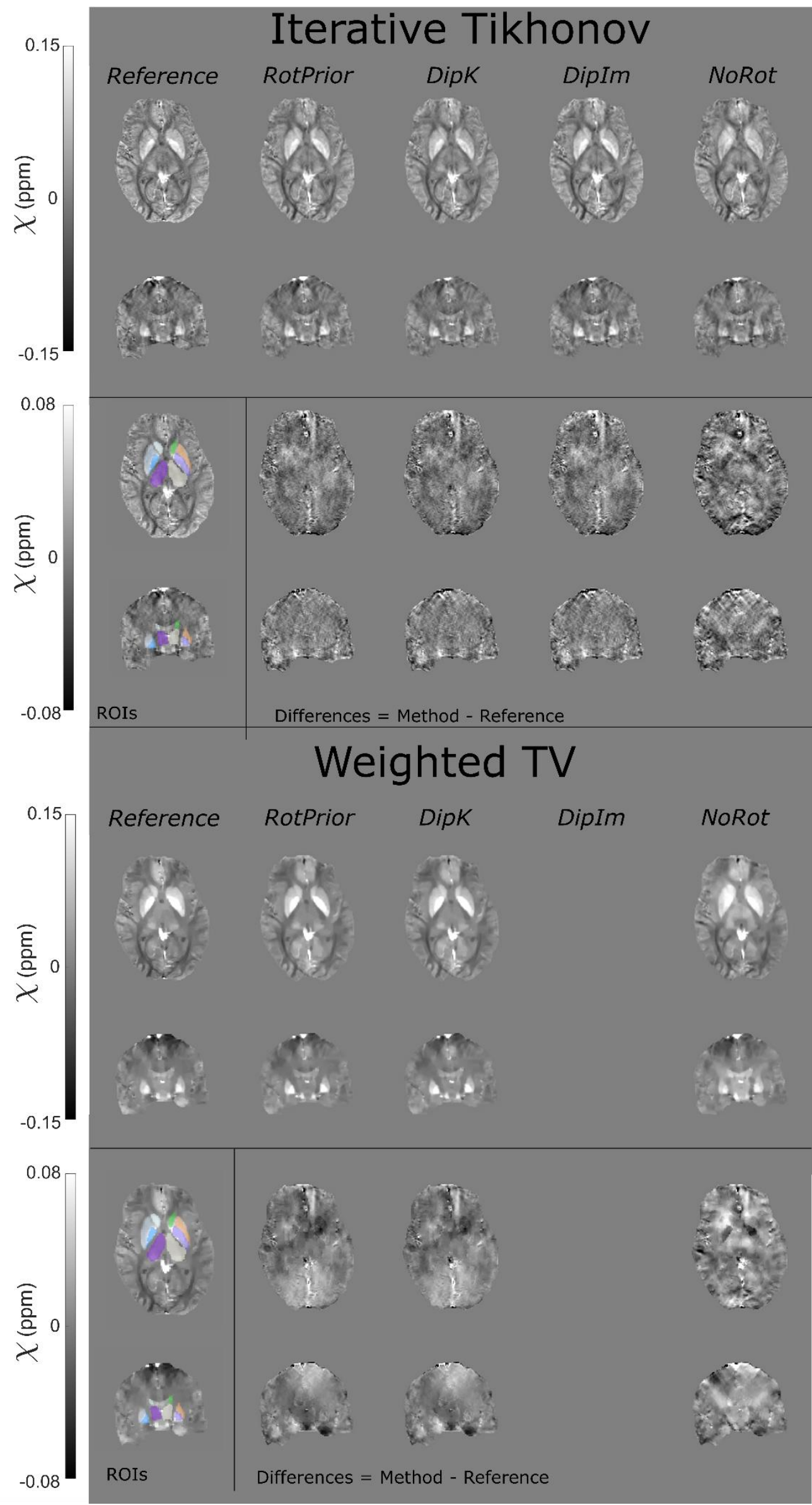


Figure 10: $\chi$ maps and difference images illustrating the effects of all tilt correction schemes on susceptibility calculation in vivo. An axial and a coronal slice are shown for a volume tilted at $-20^{\circ}$ and a reference $\left(0^{\circ}\right)$ volume with all $\chi$ maps calculated using the iterative Tikhonov method (top) and weighted linear TV (bottom). wTV with DipIm fails at non-zero angles and is therefore omitted from the figure. NoRot leads to the largest differences and image artefacts throughout the brain for iterative

Tikhonov and wTV methods. The EVE ROIs used are shown (bottom left). Results from TKD (Supporting Figure 5) are very similar.

\section{Discussion}

We have shown that oblique acquisition must be accounted for in the QSM pipeline to ensure accurate susceptibility estimates throughout the brain. For all background field removal and susceptibility calculation methods tested, if the magnetic dipole kernel is left misaligned to the $\widehat{\boldsymbol{B}}_{0}$ direction (NoRot), which is often the default mode of QSM toolboxes, then significant susceptibility errors result.

Through the analysis of the effect of tilted acquisition on a numerical phantom and one subject in vivo, we have shown that any rotations that are applied to a wrapped field map prior to phase unwrapping will result in incorrect unwrapping, using Laplacian and SEGUE unwrapping techniques, and subsequent artefacts in the resulting QSMs. Results indicate that, for PDF background field removal, rotating the image into the scanner frame and using a $k$-space dipole defined in the scanner frame (RotPrior correction method) provides the most accurate susceptibility maps. If no image rotations are desired, due to unwanted interpolation effects, LBV or V-SHARP are recommended as they are largely unaffected by oblique acquisition. Both TKD and iterative Tikhonov susceptibility calculation methods provide the most accurate results when local field maps are rotated into alignment with the scanner axes and a $k$-space dipole, defined in the scanner frame, is used (RotPrior). The same conclusion holds for weighted linear TV, but susceptibility calculation can be carried out in the oblique image frame without any rotations provided the correct $\widehat{\boldsymbol{B}}_{0}$ direction is used in defining the $k$-space dipole (DipK correction method). We therefore recommend rotating the total field map into alignment with the scanner frame after phase unwrapping but prior to background field removal.

Both the numerical phantom and in vivo results indicate that when wrapped phase images are rotated prior to phase unwrapping (with the correction scheme RotPrior), artefacts arise for both Laplacian and SEGUE unwrapping methods. This is probably due to interpolation errors along phase wraps (Figure 4). Therefore, any phase unwrapping must be carried out in images left in the same orientation as acquired, with rotations only being applied afterwards to avoid artefacts.

When using PDF for background field removal, numerical phantom and in-vivo results show that RotPrior consistently provides the most accurate susceptibility maps while NoRot performs the worst (Figures 5 and 8). Striping artefacts arise in local field maps in both the numerical phantom and in vivo when using PDF with the DipK method. First identified by Dixon, E. ${ }^{7}$, these striping artefacts 
are present due to the violations in circular continuity when defining the tilted dipole in $k$-space and using the inverse discrete Fourier transform to transform the susceptibility maps into image-space (which enforces periodicity, see Supporting Figure 6). Striping artefacts arise from regions of high susceptibility changes, such as on the brain boundaries (Figures $5 \mathrm{c}$ and $8 \mathrm{c}$ ). DipIm also resulted in poor background field removal, most likely due to fitting to the incorrect twisted or sheared unit dipole field (bottom row of Figure 1). To avoid artefacts and robustly achieve background field removal with PDF, total field maps must be rotated into alignment with the scanner frame prior to PDF background field removal as it is then possible to use the non-oblique dipole, which doesn't violate circular continuity (Supporting Figure 7). We showed that LBV and V-SHARP were mostly unaffected by oblique acquisition, with the differences between zero and non-zero tilt angles arising solely from rotation interpolation effects.

Given that RotPrior is the most accurate method for PDF, the typically necessary mask erosion must be carried out after rotation into the reference space. Artefacts that arise along the boundaries of the local field map if erosion is carried out prior to rotation (Supporting Figure 4a and 4b) probably arise from distortion of the dipolar background fields due to interpolation at the edges. In contrast, VSHARP does not show substantial differences with mask erosion before $v$. after rotation which suggests that the interpolation may not substantially affect the harmonic nature of the background fields on which this method relies.

We found TKD and iterative Tikhonov regularisation to be affected by oblique image orientation and most accurate with RotPrior. We showed Weighted linear TV to be relatively robust to oblique acquisition. For all susceptibility calculation methods tested, a unit dipole field misaligned to the main magnetic field (NoRot) leads to artefacts and substantial errors in susceptibility maps. The subtle differences between correction methods found in the phantom ROIs (Figure 6) were not apparent in vivo between $\pm 20^{\circ}$ (Figure 10) probably due to noise, motion and the expected variability in susceptibility maps over repeated acquisitions ${ }^{51,52}$.

Our results also show that, for oblique acquisition with a limited tilt angle $\left(\leq \pm 20^{\circ}\right)$ as is common in neuroimaging, DipK and RotPrior perform similarly in vivo for both background field removal and susceptibility calculation. However, our results at larger tilt angles in the numerical phantom show that DipK is less accurate than RotPrior, therefore, for certain imaging applications including cardiac imaging and pelvic imaging where large tilt angles of up to $45^{\circ}$ are often required, tilt correction is likely to be essential for accurate susceptibility mapping.

Therefore, we recommend accounting for oblique acquisition by using the RotPrior tiltcorrection method before background field removal since this method gave the most accurate susceptibility maps in both the numerical phantom and in vivo. If desired, the susceptibility map can be rotated back into the original orientation after susceptibility calculation to facilitate comparison with other (processed) images. It is possible to build an alternative rotation-free pipeline of methods relatively unaffected by oblique acquisition (such as LBV and weighted non-linear TV) but those 
methods must be checked to ensure true independence of image orientation. However, such an approach limits the choice of methods for the steps in the QSM pipeline, which could lead to suboptimal susceptibility maps. For example, LBV's highly specific boundary approximations can be easily violated making it easier to simply rotate the field maps in some cases. These aspects must be considered carefully when designing a QSM pipeline.

\section{Conclusions}

Oblique acquisition must be accounted for in the QSM pipeline to avoid artefacts and erroneous susceptibility estimates. We recommend rotating the total field map into alignment with the scanner frame after phase unwrapping but before background field removal (and then rotating the final susceptibility map back into the original orientation). Alternatively, a QSM pipeline relatively robust to oblique acquisition can be built from a more limited number of image-orientation-independent methods (e.g. LBV or V-SHARP for background field removal and weighted non-linear TV for susceptibility calculation). However, care must be taken in weighing up the minimal effects of image interpolation (from tilt correction rotations) versus choosing from a smaller range of methods that are orientation independent, as they may not be as accurate nor optimal for a given data set. It would also be vital to ensure a chosen method is independent of slice orientation, which may require further investigation. Our recommended correction scheme ensures that all methods developed for each stage of the QSM pipeline can be used and optimised.

We provide an open-source MATLAB function that can be easily incorporated into existing MATLAB-based QSM pipelines and used both to align oblique 3D volumes with the scanner frame and to rotate image volumes (e.g., susceptibility maps) back into the original oblique image orientation. This function is freely available at: https://github.com/o-snow/QSM_TiltCorrection.git. Our results allow accurate susceptibility maps to be obtained from oblique image acquisitions. This is an important step in translating QSM into clinical practice.

\section{Acknowledgments}

Oliver Kiersnowski's work was supported by the EPSRC-funded UCL Centre for Doctoral Training in Intelligent, Integrated Imaging in Healthcare (i4health) (EP/S021930/1). John Thornton received support from the National Institute for Health Research University College London Hospitals Biomedical Research Centre. Karin Shmueli and Anita Karsa were supported by European Research Council Consolidator Grant DiSCo MRI SFN 770939. We would like to thank Carlos Milovic for his help with the FANSI toolbox, and the healthy volunteer for their time. 


\section{Supporting Information Figures}
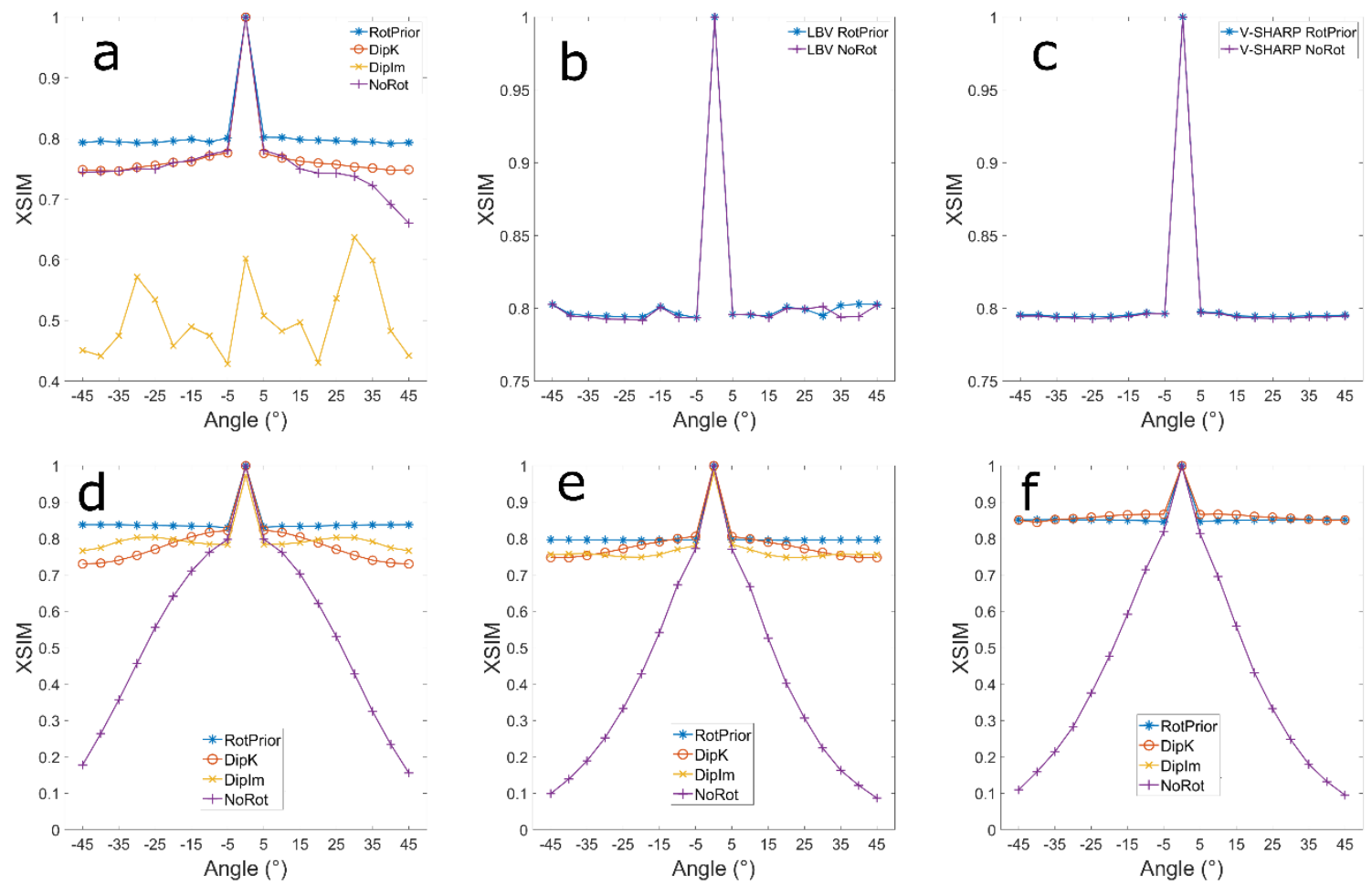

Supporting Figure S1: Results in the numerical phantom from oblique image volumes tilted about the $\mathrm{x}$-axis. The volumes were padded to ensure no parts of the original image volume were cut off during rotations. XSIM measurements of QSM calculated with tilt corrections prior to background field removal with PDF (a), LBV (b) and V-SHARP (c) agree with unpadded results (Figure 5). XSIM measurements comparing tilt correction schemes prior to susceptibility calculation with TKD (d), iterative Tikhonov regularisation (e) and linear weighted TV (f) methods are also in agreement with unpadded results (Figure 6, bottom row). 

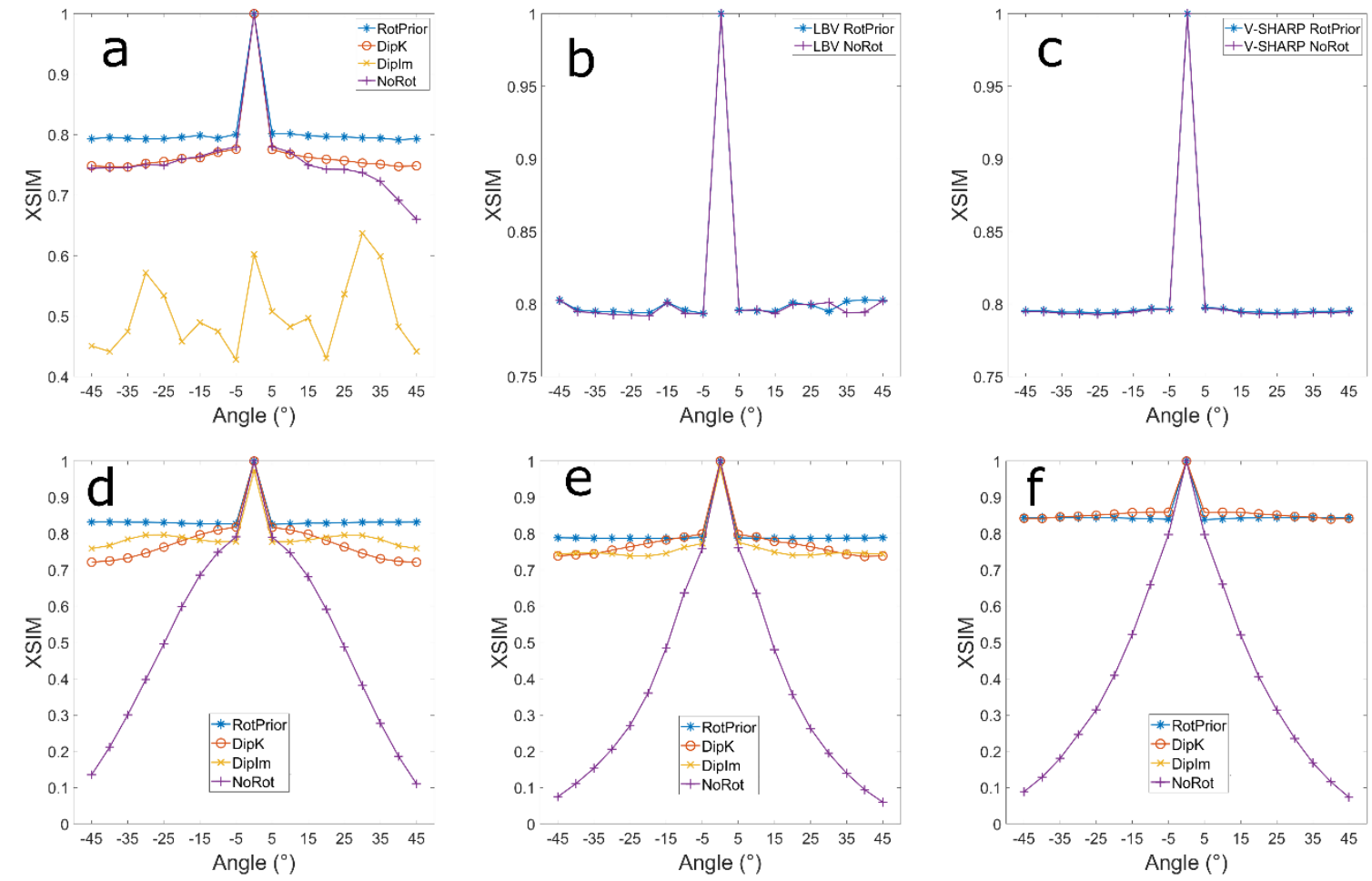

Supporting Figure S2: Results in the numerical phantom from oblique image volumes tilted about the $y$-axis with padded image volumes to ensure no parts of the original image volume were cut off during rotations. XSIM measurements of QSMs calculated with tilt corrections prior to background field removal with PDF (a), LBV (b) and V-SHARP (c) agree with unpadded results and rotations about the $\mathrm{x}$-axis (Figure 5, Supporting Figure 1a,b,c). XSIM measurements comparing tilt correction schemes prior to susceptibility calculation with TKD (d), iterative Tikhonov regularisation (e) and linear weighted TV (f) methods are also in agreement with unpadded results and $\mathrm{x}$-axis rotations (Figure 6, bottom row; Supporting figure $1 \mathrm{~d}, \mathrm{e}, \mathrm{f}$ ). 

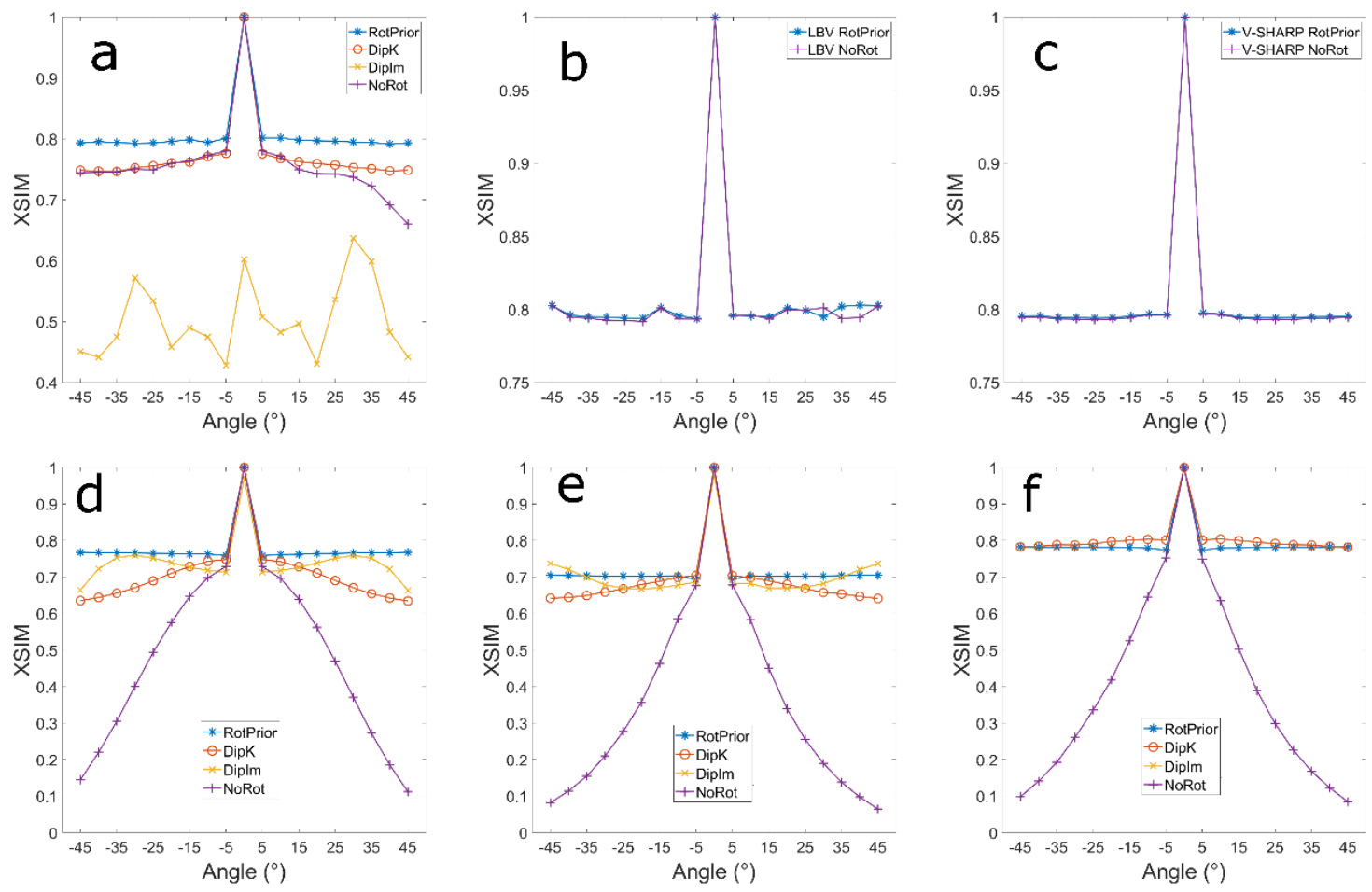

Supporting Figure S3: numerical phantom results from rotations about the $\mathrm{y}=\mathrm{x}$ axis with padded image volumes to ensure no parts of the original image volume was cut off during rotations. XSIM measurements of QSMs during the background field removal part of the pipeline for PDF (a), LBV

(b) and V-SHARP (c) agree with unpadded results and rotations about the $\mathrm{x}$ and $\mathrm{y}$-axes. XSIM measurements comparing susceptibility calculation methods TKD (d), iterative Tikhonov regularisation (e) and linear weighted TV ( $\mathrm{f}$ ) are also in agreement with unpadded results and $\mathrm{x}$ and $\mathrm{y}$ axis rotations (Figure 6, bottom row; supporting figures 1 and $2 \mathrm{~d}, \mathrm{e}, \mathrm{f}$ ). 


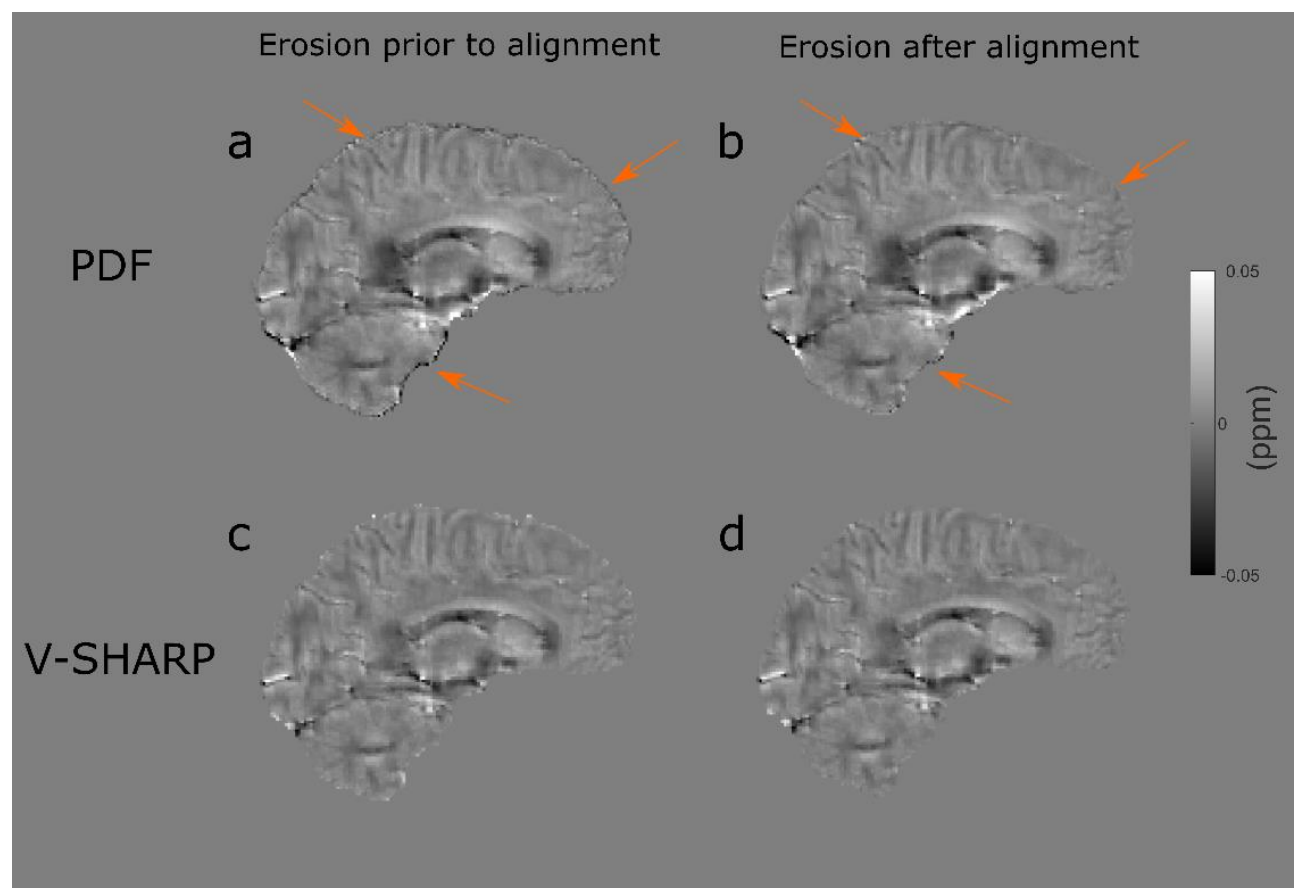

Supporting Figure S4: For improved PDF performance, the brain mask is typically eroded. If this erosion takes place prior to rotating the field map into alignment with $\hat{B}_{0}$ (a) compared to after (b), artefacts arise along the edges of the local field map following background field removal with PDF (a, orange arrows), increasing the RMSE and decreasing the XSIM. These artefacts do not arise when using V-SHARP (c: erosion before, d: erosion after). 


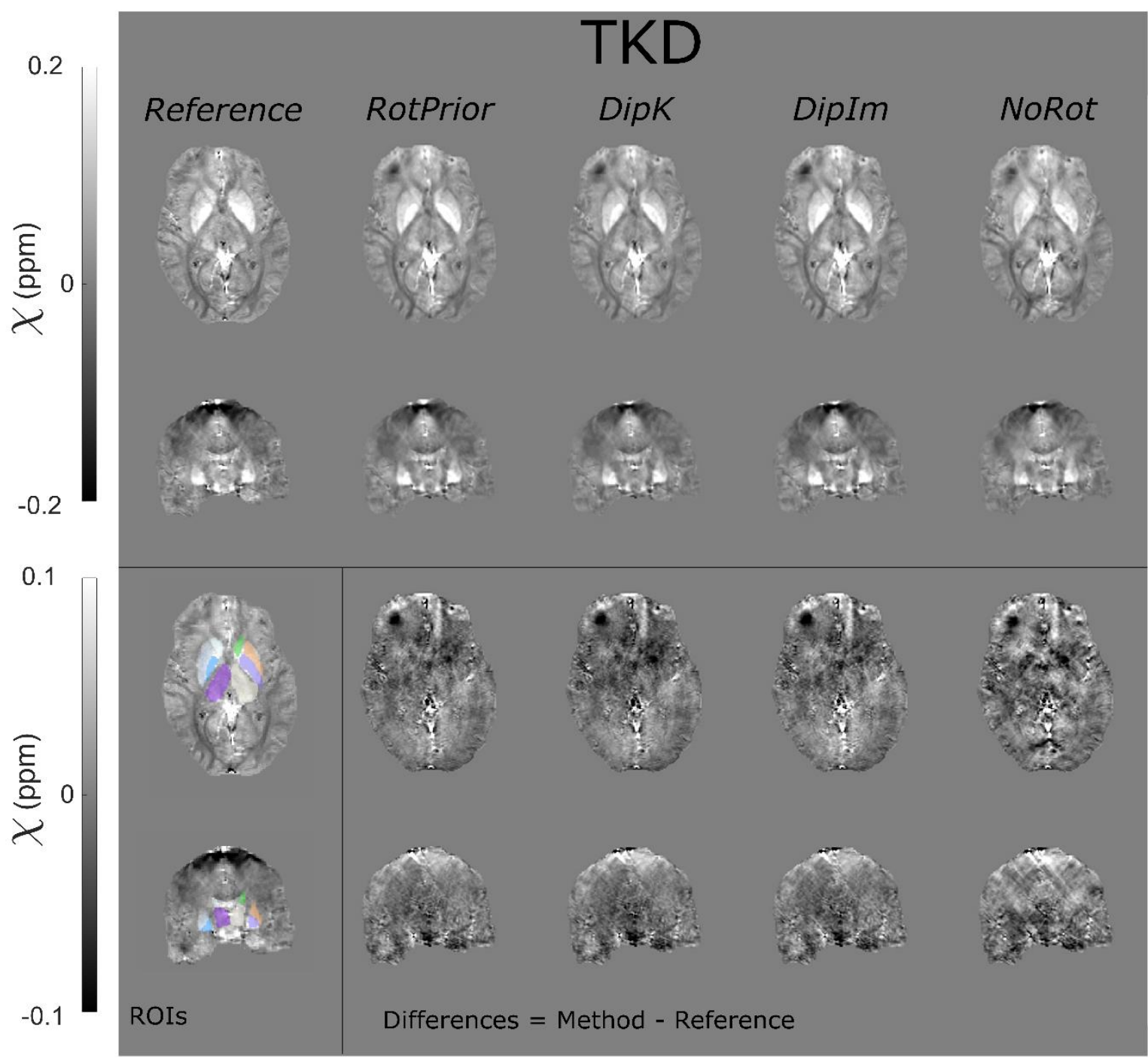

Supporting Figure S5: $\chi$ maps and difference images illustrating the effects of all tilt correction schemes on susceptibility calculation in vivo. An axial and a coronal slice are shown for a volume tilted at $-20^{\circ}$ and a reference $\left(0^{\circ}\right)$ volume with all $\chi$ maps calculated using Thresholded k-space (TKD) method. NoRot leads to the largest differences and image artefacts throughout the brain. The EVE ROIs used are shown (bottom left). These results are very similar to iterative Tikhonov and weighted linear TV susceptibility maps (Figure 10). 
bioRxiv preprint doi: https://doi org/10.1101/2021.1130.470544; this version posted December 1, 2021. The copyright holder for this preprint (which was not certified by peer review) is the author/funder, who has granted bioRxiv a license to display the preprint in perpetuity. It is made available under aCC-BY-ND 4.0 International license.

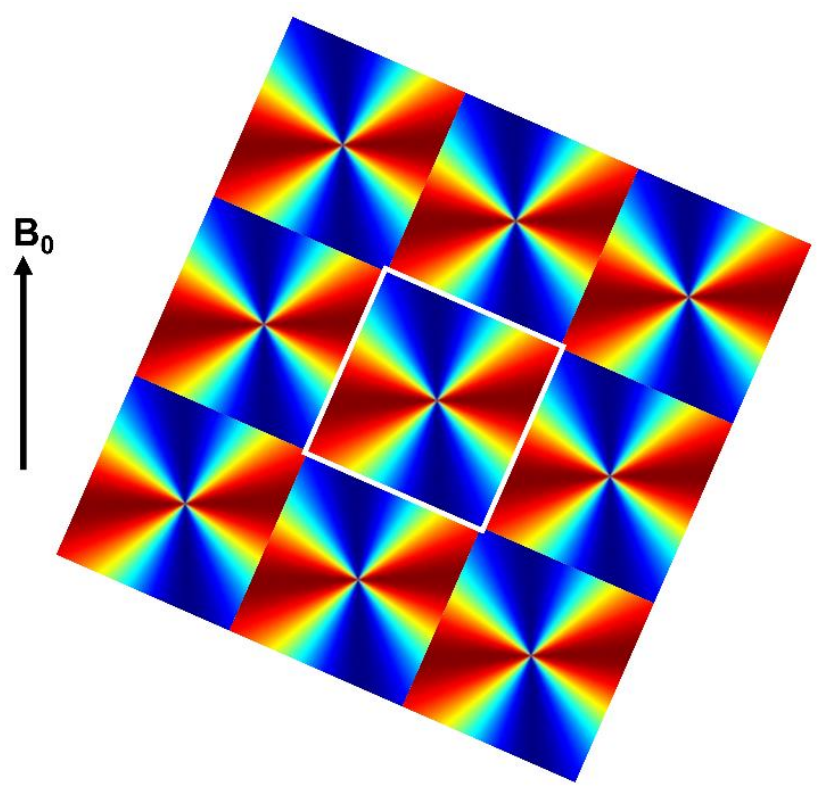

Supporting Figure S6: Oblique k-space magnetic dipole kernels laid side by side to illustrate the violations in circular continuity. These dipoles are used in the DipK correction method, which leads to striping artifacts due to the violations in circular continuity i.e. discontinuities at the boundaries of the rotated k-space dipoles (white square and arrows). 


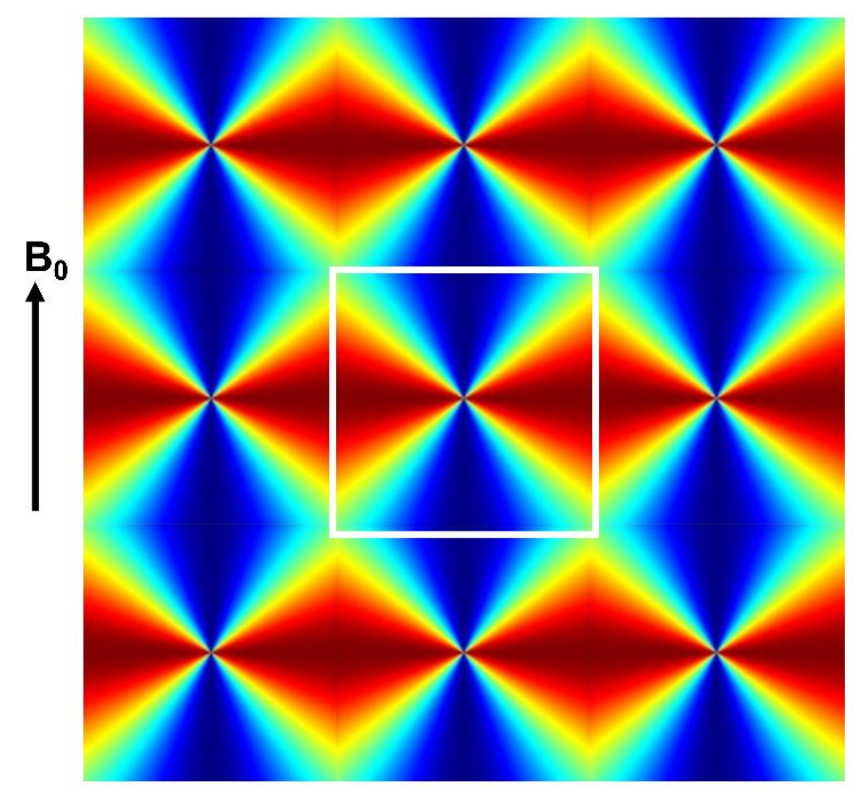

Supporting Figure S7: Non-oblique k-space magnetic dipole kernels laid side by side to illustrate circular continuity. When there is no oblique acquisition then there are no violations in circular continuity i.e. identical values and no discontinuities at the boundaries of the k-space dipoles (white square). 


\section{References}

1. Traboulsee A, Simon JH, Stone L, et al. Revised recommendations of the consortium of MS centers task force for a standardized MRI protocol and clinical guidelines for the diagnosis and follow-up of multiple sclerosis. American Journal of Neuroradiology. 2016;37(3):394-401. doi:10.3174/ajnr.A4539

2. Jack CR, Theodore WH, Cook M, McCarthy G. MRI-based hippocampal volumetrics: Data acquisition, normal ranges, and optimal protocol. Magnetic Resonance Imaging. 1995;13(8):1057-1064. doi:10.1016/0730-725X(95)02013-J

3. Chen W, Zhu XH. Suppression of physiological eye movement artifacts in functional MRI using slab presaturation. Magnetic Resonance in Medicine. 1997;38(4):546-550. doi:10.1002/mrm.1910380407

4. Deistung A, Schweser F, Reichenbach JR. Overview of quantitative susceptibility mapping. NMR in Biomedicine. 2017;30(4). doi:10.1002/nbm.3569

5. Wang Y, Liu T. Quantitative susceptibility mapping (QSM): Decoding MRI data for a tissue magnetic biomarker. Magnetic Resonance in Medicine. 2015;73(1):82-101. doi:10.1002/mrm.25358

6. Shmueli K. Quantitative Susceptibility Mapping. In: Quantitative Magnetic Resonance Imaging. 1st ed. Elsevier; 2020.

7. Dixon EC. Applications of MRI Magnetic Susceptibility Mapping in PET-MRI Brain Studies. Doctoral thesis, UCL (University College London). 2018.

8. Kiersnowski OC, Karsa A, Thornton JS, Shmueli K. The Effect of Oblique Image Slices on the Accuracy of Quantitative Susceptibility Mapping and a Robust Tilt Correction Method \#0794. Proceedings of the International Society for Magnetic Resonance in Medicine. 2021. doi:10.1002/mrm.22135

9. Eskreis-Winkler S, Zhang Y, Zhang J, et al. The clinical utility of QSM: disease diagnosis, medical management, and surgical planning. NMR in Biomedicine. 2017;30(4). doi:10.1002/nbm.3668

10. Liu C, Wei H, Gong N jie, Cronin M, Dibb R, Decker K. Quantitative Susceptibility Mapping: Contrast Mechanisms and Clinical Applications. Tomography. 2015;1(1):3-17. doi:10.18383/j.tom.2015.00136

11. Haacke EM, Liu S, Buch S, Zheng W, Wu D, Ye Y. Quantitative susceptibility mapping: Current status and future directions. Magnetic Resonance Imaging. 2015;33(1):1-25. doi:10.1016/j.mri.2014.09.004

12. Liu C, Li W, Tong KA, Yeom KW, Kuzminski S. Susceptibility-weighted imaging and quantitative susceptibility mapping in the brain. Journal of Magnetic Resonance Imaging. 2015;42(1):23-41. doi:10.1002/jmri.24768

13. Bray TJP, Karsa A, Bainbridge A, et al. Association of bone mineral density and fat fraction with magnetic susceptibility in inflamed trabecular bone. Magnetic Resonance in Medicine. 2019;81(5):3094-3107. doi:10.1002/mrm.27634

14. MEDI Toolbox. http://pre.weill.cornell.edu/mri/pages/qsm.html. 
15. Neuroimaging Informatics Technology Initiative. https://nifti.nimh.nih.gov/.

16. Jenkinson M, Beckmann CF, Behrens TEJ, Woolrich MW, Smith SM. FSL. NeuroImage. 2012;62:782-790. doi:10.1016/j.neuroimage.2011.09.015

17. Marques JP, Meineke J, Milovic C, et al. QSM reconstruction challenge 2.0: A realistic in silico head phantom for MRI data simulation and evaluation of susceptibility mapping procedures. Magnetic Resonance in Medicine. 2021;86(1):526-542. doi:10.1002/mrm.28716

18. Liu T, Wisnieff C, Lou M, Chen W, Spincemaille P, Wang Y. Nonlinear formulation of the magnetic field to source relationship for robust quantitative susceptibility mapping. Magnetic Resonance in Medicine. 2013;69(2):467-476. doi:10.1002/mrm.24272

19. Biondetti E, Karsa A, Thomas DL, Shmueli K. Investigating the accuracy and precision of TEdependent versus multi-echo QSM using Laplacian-based methods at 3 T. Magnetic Resonance in Medicine. 2020;84(6):3040-3053. doi:10.1002/mrm.28331

20. Shmueli K, De Zwart JA, Van Gelderen P, Li TQ, Dodd SJ, Duyn JH. Magnetic susceptibility mapping of brain tissue in vivo using MRI phase data. Magnetic Resonance in Medicine. 2009;62(6):1510-1522. doi:10.1002/mrm.22135

21. MRI susceptibility calculation methods. https://xip.uclb.com/i/software/mri_qsm_tkd.html.

22. Schweser F, Deistung A, Sommer K, Reichenbach JR. Toward online reconstruction of quantitative susceptibility maps: Superfast dipole inversion. Magnetic Resonance in Medicine. 2013;69(6):1581-1593. doi:10.1002/mrm.24405

23. Karsa A, Punwani S, Shmueli K. An optimized and highly repeatable MRI acquisition and processing pipeline for quantitative susceptibility mapping in the head-and-neck region. Magnetic Resonance in Medicine. 2020;84(6):3206-3222. doi:10.1002/mrm.28377

24. MRI susceptibility calculation methods. https://xip.uclb.com/i/software/mri_qsm_tkd.html.

25. Chary K, Nissi MJ, Nykänen O, et al. Quantitative susceptibility mapping of the rat brain after traumatic brain injury. NMR in Biomedicine. 2021;34(2):1-15. doi:10.1002/nbm.4438

26. Stone AJ, Tornifoglio B, Johnston RD, Shmueli K, Kerskens C, Lally C. Quantitative susceptibility mapping of carotid arterial tissue ex vivo: Assessing sensitivity to vessel microstructural composition. Magnetic Resonance in Medicine. 2021;(February):2512-2527. doi:10.1002/mrm.28893

27. Berg RC, Preibisch C, Thomas DL, Shmueli K, Biondetti E. Investigating the effect of flow compensation and quantitative susceptibility mapping method on the accuracy of venous susceptibility measurement. NeuroImage. 2021;240(March):118399. doi:10.1016/j.neuroimage.2021.118399

28. Hansen PC, O'Leary DP. The Use of the L-Curve in the Regularization of Discrete Ill-Posed Problems. SIAM Journal on Scientific Computing. 1993;14(6):1487-1503. doi:10.1137/0914086

29. FANSI Toolbox. https://gitlab.com/cmilovic/FANSI-toolbox.

30. Milovic C, Bilgic B, Zhao B, Acosta-Cabronero J, Tejos C. Fast nonlinear susceptibility inversion with variational regularization. Magnetic Resonance in Medicine. 2018;80(2):814821. doi:10.1002/mrm. 27073 
bioRxiv preprint doi: https://doi org/10.1101/2021.1130.470544: this version posted December 1,2021 . The copyright holder for this preprint (which was not certified by peer review) is the author/funder, who has granted bioRxiv a license to display the preprint in perpetuity. It is made available under aCC-BY-ND 4.0 International license.

31. Bilgic B, Langkammer C, Marques JP, Meineke J, Milovic C, Schweser F. QSM reconstruction challenge 2.0: Design and report of results. Magnetic Resonance in Medicine. 2021;86(3):1241-1255. doi:10.1002/mrm.28754

32. Milovic C, Tejos C, Irarrazaval P. Structural Similarity Index Metric setup for QSM applications (XSIM). In: 5th International Workshop on MRI Phase Contrast \& Quantitative Susceptibility Mapping. Seoul, Korea; 2019.

33. Marques JP, Bowtell RW. Using forward calculations of the magnetic field perturbation due to a realistic vascular model to explore the BOLD effect. NMR in Biomedicine. 2008. doi:10.1002/nbm. 1224

34. Salomir R, De Senneville BD, Moonen CTW. A fast calculation method for magnetic field inhomogeneity due to an arbitrary distribution of bulk susceptibility. Concepts in Magnetic Resonance Part B: Magnetic Resonance Engineering. 2003. doi:10.1002/cmr.b.10083

35. Buch S, Liu S, Ye Y, Cheng YCN, Neelavalli J, Haacke EM. Susceptibility mapping of air, bone, and calcium in the head. Magnetic Resonance in Medicine. 2015;73(6):2185-2194. doi: $10.1002 / \mathrm{mrm} .25350$

36. Burgos N, Cardoso MJ, Thielemans K, et al. Attenuation correction synthesis for hybrid PETMR scanners: Application to brain studies. IEEE Transactions on Medical Imaging. 2014;33(12):2332-2341. doi:10.1109/TMI.2014.2340135

37. NiftyWeb. http://niftyweb.cs.ucl.ac.uk/.

38. Prados F, Cardoso MJ, Burgos N, et al. NiftyWeb: web based platform for image processing on the cloud. 24th Scientific Meeting and Exhibition of the International Society for Magnetic Resonance in Medicine (ISMRM). 2016;(May):1049.

39. Schweser F, Robinson SD, de Rochefort L, Li W, Bredies K. An illustrated comparison of processing methods for phase MRI and QSM: removal of background field contributions from sources outside the region of interest. NMR in Biomedicine. 2017;30(4). doi:10.1002/nbm.3604

40. Liu T, Khalidov I, de Rochefort L, et al. A novel background field removal method for MRI using projection onto dipole fields (PDF). NMR in Biomedicine. 2011;24(9):1129-1136. doi:10.1002/nbm. 1670

41. Zhou D, Liu T, Spincemaille P, Wang Y. Background field removal by solving the Laplacian boundary value problem. NMR in Biomedicine. 2014;27(3):312-319. doi:10.1002/nbm.3064

42. $\mathrm{Li} \mathrm{W}, \mathrm{Wu}$ B, Liu C. Quantitative susceptibility mapping of human brain reflects spatial variation in tissue composition. NeuroImage. 2011;55:1645-1656.

doi:10.1016/j.neuroimage.2010.11.088

43. Schofield MA, Zhu Y. Fast phase unwrapping algorithm for interferometric applications. Optics Letters. 2003. doi:10.1364/ol.28.001194

44. Karsa A, Shmueli K. SEGUE: A Speedy rEgion-Growing Algorithm for Unwrapping Estimated Phase. IEEE Transactions on Medical Imaging. 2019;38(6):1347-1357. doi:10.1109/TMI.2018.2884093

45. Eckstein K, Dymerska B, Bachrata B, et al. Computationally Efficient Combination of Multichannel Phase Data From Multi-echo Acquisitions (ASPIRE). Magnetic Resonance in Medicine. 2018;79(6):2996-3006. doi:10.1002/mrm.26963 
bioRxiv preprint doi: https://doi.org/10.1101/2021.1130.470544: this version posted December 1,2021 . The copyright holder for this preprint (which was not certified by peer review) is the author/funder, who has granted bioRxiv a license to display the preprint in perpetuity. It is made available under aCC-BY-ND 4.0 International license.

46. Liu T, Wisnieff C, Lou M, Chen W, Spincemaille P, Wang Y. Nonlinear formulation of the magnetic field to source relationship for robust quantitative susceptibility mapping. Magnetic Resonance in Medicine. 2013;69(2):467-476. doi:10.1002/mrm.24272

47. Smith SM. Fast robust automated brain extraction. Human Brain Mapping. 2002;17(3):143155. doi:10.1002/hbm.10062

48. Modat M, Cash DM, Daga P, Winston GP, Duncan JS, Ourselin S. Global image registration using a symmetric block-matching approach. Journal of Medical Imaging. 2014;1(2):024003. doi:10.1117/1.jmi.1.2.024003

49. Schweser F, Deistung A, Reichenbach JR. Foundations of MRI phase imaging and processing for Quantitative Susceptibility Mapping (QSM). Zeitschrift fur Medizinische Physik. 2016;26(1):6-34. doi:10.1016/j.zemedi.2015.10.002

50. Lim IAL, Faria A V, Li X, et al. Human brain atlas for automated region of interest selection in quantitative susceptibility mapping: application to determine iron content in deep gray matter structures. NeuroImage. 2013;82:449-469. doi:10.1016/j.neuroimage.2013.05.127

51. Feng X, Deistung A, Reichenbach JR. Quantitative susceptibility mapping (QSM) and R2* in the human brain at $3 \mathrm{~T}$ : Evaluation of intra-scanner repeatability. Zeitschrift fur Medizinische Physik. 2018;28(1):36-48. doi:10.1016/j.zemedi.2017.05.003

52. Karsa A, Punwani S, Shmueli K. An optimized and highly repeatable MRI acquisition and processing pipeline for quantitative susceptibility mapping in the head-and-neck region. Magnetic Resonance in Medicine. 2020;84(6):3206-3222. doi:10.1002/mrm.28377 